\title{
Measuring the tensor to scalar ratio from CMB B-modes in the presence of foregrounds ${ }^{\star}$
}

\author{
M. Betoule ${ }^{1}$, E. Pierpaoli ${ }^{2}$, J. Delabrouille ${ }^{1}$, M. Le Jeune ${ }^{1}$, and J.-F. Cardoso ${ }^{1}$ \\ 1 AstroParticule et Cosmologie (APC), CNRS: UMR 7164, Université Denis Diderot, Paris 7, Observatoire de Paris, France \\ e-mail: betoule@apc.univ-paris7.fr \\ 2 University of Southern California, Los Angeles, CA 90089-0484, USA
}

Received 7 January 2009 / Accepted 7 May 2009

\section{ABSTRACT}

\begin{abstract}
Aims. We investigate the impact of polarised foreground emission on the performances of future CMB experiments aiming to detect primordial tensor fluctuations in the early universe. In particular, we study the accuracy that can be achieved in measuring the tensorto-scalar ratio $r$ in the presence of foregrounds.

Methods. We designed a component separation pipeline, based on the SMICA method, aimed at estimating $r$ and the foreground contamination from the data with no prior assumption on the frequency dependence or spatial distribution of the foregrounds. We derived error bars accounting for the uncertainty on foreground contribution. We used the current knowledge of galactic and extragalactic foregrounds as implemented in the Planck sky model (PSM) to build simulations of the sky emission. We applied the method to simulated observations of this modelled sky emission, for various experimental setups. Instrumental systematics are not considered in this study.

Results. Our method, with Planck data, permits us to detect $r=0.1$ from B-modes only at more than $3 \sigma$. With a future dedicated space experiment, such as EPIC, we can measure $r=0.001$ at $\sim 6 \sigma$ for the most ambitious mission designs. Most of the sensitivity to $r$ comes from scales $20 \leq \ell \leq 150$ for high $r$ values, shifting to lower $\ell$ 's for progressively smaller $r$. This shows that large-scale foreground emission does not prevent proper measurement of the reionisation bump for full sky experiments. We also investigate the observation of a small but clean part of the sky. We show that diffuse foregrounds remain a concern for a sensitive ground-based experiment with a limited frequency coverage when measuring $r<0.1$. Using the Planck data as additional frequency channels to constrain the foregrounds in such ground-based observations reduces the error by a factor two but does not allow detection of $r=0.01$. An alternate strategy, based on a deep field space mission with a wide frequency coverage, would allow us to deal with diffuse foregrounds efficiently, but is in return quite sensitive to lensing contamination. In contrast, we show that all-sky missions are nearly insensitive to small-scale contamination (point sources and lensing) if the statistical contribution of such foregrounds can be modelled accurately. Our results do not significantly depend on the overall level and frequency dependence of the diffused foreground model, when varied within the limits allowed by current observations.
\end{abstract}

Key words. cosmology: cosmic microwave background - cosmology: cosmological parameters - cosmology: observations

\section{Introduction}

After the success of the WMAP space mission in mapping the cosmic microwave background (CMB) temperature anisotropies, much attention now turns towards the challenge of measuring CMB polarisation, in particular pseudo-scalar polarisation modes (the B-modes) of primordial origin. These B-modes offer one of the best options for constraining inflationary models (Seljak \& Zaldarriaga 1997; Hu \& White 1997; Kamionkowski et al. 1997; Kamionkowski \& Kosowsky 1998; Baumann \& Peiris 2008).

First polarisation measurements have already been obtained by a number of instruments (Kovac et al. 2002; Sievers \& CBI Collaboration 2005; Page et al. 2007), but no detection of Bmodes has been claimed yet. While several ground-based and balloon-borne experiments are already operational, or under construction, no CMB-dedicated space-mission is planned after Planck at the present time: whether there should be one for CMB B-modes, and how it should be designed, are still open questions.

* Appendices are only available in electronic form at http://www . aanda.org
As CMB polarisation anisotropies are expected to be significantly smaller than temperature anisotropies (a few per cent at most), improving detector sensitivities is the first major challenge towards measuring CMB polarisation B-modes. It is not, however, the only one. Foreground emissions from the galactic interstellar medium (ISM) and from extra-galactic objects (galaxies and clusters of galaxies) superimpose to the CMB. Most foregrounds are expected to emit polarised light, with a polarisation fraction typically comparable to or larger than that of the CMB. Component separation (disentangling CMB emission from all these foregrounds) is needed to extract cosmological information from observed frequency maps. The situation is particularly severe for the B-modes of CMB polarisation, which will be, if measurable, subdominant on every scale and at every frequency.

The main objective of this paper is to evaluate the accuracy with which various upcoming or planned experiments can measure the tensor-scalar ratio $r$ (see Peiris et al. (2003) for a precise definition) in the presence of foregrounds. This problem has been addressed before: Tucci et al. (2005) investigate the lower bound for $r$ that can be achieved considering a simple foreground cleaning technique, based on the extrapolation of 
foreground templates and subtraction from a channel dedicated to CMB measurement; Verde et al. (2006) assume foreground residuals at a known level in a cleaned map, treat them as additional Gaussian noise, and compute the error on $r$ due to such excess noise; Amblard et al. (2007) investigate how best to select the frequency bands of an instrument and how to distribute a fixed number of detectors among them, to maximally reject galactic foreground contamination. This analysis is based on an internal linear combination cleaning technique similar to the one of Tegmark et al. (2003) on WMAP temperature anisotropy data. The last two studies assume somehow that the residual contamination level is perfectly known - information that is used to derive error bars on $r$.

In this paper, we relax this assumption and propose a method for estimating the uncertainty on residual contamination from the data themselves, as would be the case for real data analysis. We test our method on semi-realistic simulated data sets, including CMB and realistic foreground emission, as well as simple instrumental noise. We study a variety of experimental designs and foreground mixtures. Alternative approaches to the same question are also presented in Dunkley et al. (2008a) and Baumann et al. (2008).

This paper is organised as follows: the next section (Sect. 2) deals with polarised foregrounds and presents the galactic emission model used in this work. In Sect. 3, we propose a method, using the most recent version of the SMICA component separation framework (Cardoso et al. 2008), for providing measurements of the tensor to scalar ratio in the presence of foregrounds. In Sect. 4, we present the results obtained by applying the method to various experimental designs. Section 5 discusses the reliability of the method (and of our conclusions) against various issues, in particular modelling uncertainty. Main results are summarised in Sect. 6.

\section{Modelling polarised sky emission}

Several processes contribute to the total sky emission in the frequency range of interest for $\mathrm{CMB}$ observation (typically between 30 and $300 \mathrm{GHz}$ ). Foreground emission arises from the galactic interstellar medium (ISM), from extra-galactic objects, and from distortions of the CMB itself through its interaction with structures in the nearby universe. Although the physical processes involved and the general emission mechanisms are mostly understood, specifics of these polarised emissions in the millimetre range remain poorly known as few actual observations, on a significant enough part of the sky, have been made.

Diffuse emission from the ISM arises through synchrotron emission from energetic electrons, through free-free emission, and through grey-body emission of a population of dust grains. Small spinning dust grains with a dipole electric moment may also emit significantly in the radio domain (Draine \& Lazarian 1998). Among those processes, dust and synchrotron emissions are thought to be significantly polarised. Galactic emission also includes contributions from compact regions such as supernovae remnants and molecular clouds, which have specific emission properties.

Extra-galactic objects emit via a number of different mechanisms, each of them having its own spectral energy distribution and polarisation properties.

Finally, the CMB polarisation spectra are modified by the interactions of the CMB photons on their way from the last scattering surface. Reionisation, in particular, re-injects power in polarisation on large scales by late-time scattering of CMB photons. This produces a distinctive feature, the reionisation bump, in the
CMB B-mode spectrum at low $\ell$. Other interactions with the latter universe, and in particular lensing, contribute to hinder the measurement of the primordial signal. The lensing effect is particularly important on smaller scales as it converts a part of the dominant E-mode power into B-mode.

In the following, we review the identified polarisation processes and detail the model used for the present work, with a special emphasis on B-modes. We also discuss main sources of uncertainty in the model, as a basis for evaluating their impact on the conclusions of this paper.

Our simulations ${ }^{1}$ are based on the PSM, a sky emission simulation tool developed by the Planck collaboration for prelaunch preparation of Planck data analysis (Delabrouille et al. 2009). Figure 1 gives an overview of foregrounds as included in our baseline model. Diffuse galactic emission from synchrotron and dust dominates at all frequencies and on all scales, with a minimum (relative to CMB) between 60 and $80 \mathrm{GHz}$, depending on the galactic cut. Contaminations by lensing and a point source background are lower than primordial CMB for $r>0.01$ and for $\ell<100$, but should clearly be taken into account in attempts to measure $r<0.01$.

\subsection{Synchrotron}

Cosmic ray electrons spiralling in the galactic magnetic field produce highly polarised synchrotron emission (e.g. Rybicki \& Lightman 1979). This is the dominant contaminant of the polarised CMB signal at low frequency $(\$ 80 \mathrm{GHz})$, as can be seen in the right panel of Fig. 1. In the frequency range of interest for CMB observations, measurements of this emission have been provided, both in temperature and polarisation, by WMAP (Page et al. 2007; Gold et al. 2008). The intensity of the synchrotron emission depends on the cosmic ray density $n_{e}$, and on the strength of the magnetic field perpendicularly to the line of sight. Its frequency scaling and its intrinsic polarisation fraction $f_{s}$ depend on the energy distribution of the cosmic rays.

\subsubsection{Synchrotron emission law}

For electron density following a power law of index $p, n_{e}(E) \propto$ $E^{-p}$, the synchrotron frequency dependence is also a power law, of index $\beta_{s}=-(p+3) / 2$. So, given the intensity of the synchrotron emission at a reference frequency $v_{0}$, the intensity at the frequency $v$ reads:

$S(v)=S\left(v_{0}\right)\left(v / v_{0}\right)^{\beta_{s}}$

where the spectral index, $\beta_{s}$, is equal to -3 for a typical value $p=3$.

The synchrotron spectral index depends significantly on cosmic ray properties. It varies with the direction of the sky, and possibly, with the frequency of observation (see e.g. Strong et al. 2007 , for a review of propagation and interaction processes of cosmic rays in the galaxy). For a multi-channel experiment, the consequence of this is a decrease of the coherence of the synchrotron emission across channels, i.e. the correlation between the synchrotron emission in the various frequency bands of observation will be below unity.

Observational constraints have been put on the synchrotron emission law. A template of synchrotron emission intensity at $408 \mathrm{MHz}$ has been provided by Haslam et al. (1982). Combining

\footnotetext{
1 Foreground maps used specifically for this work are available at http://wwW . apc.univ-paris7.fr/ betoule/doku.php? id=bmodes
} 

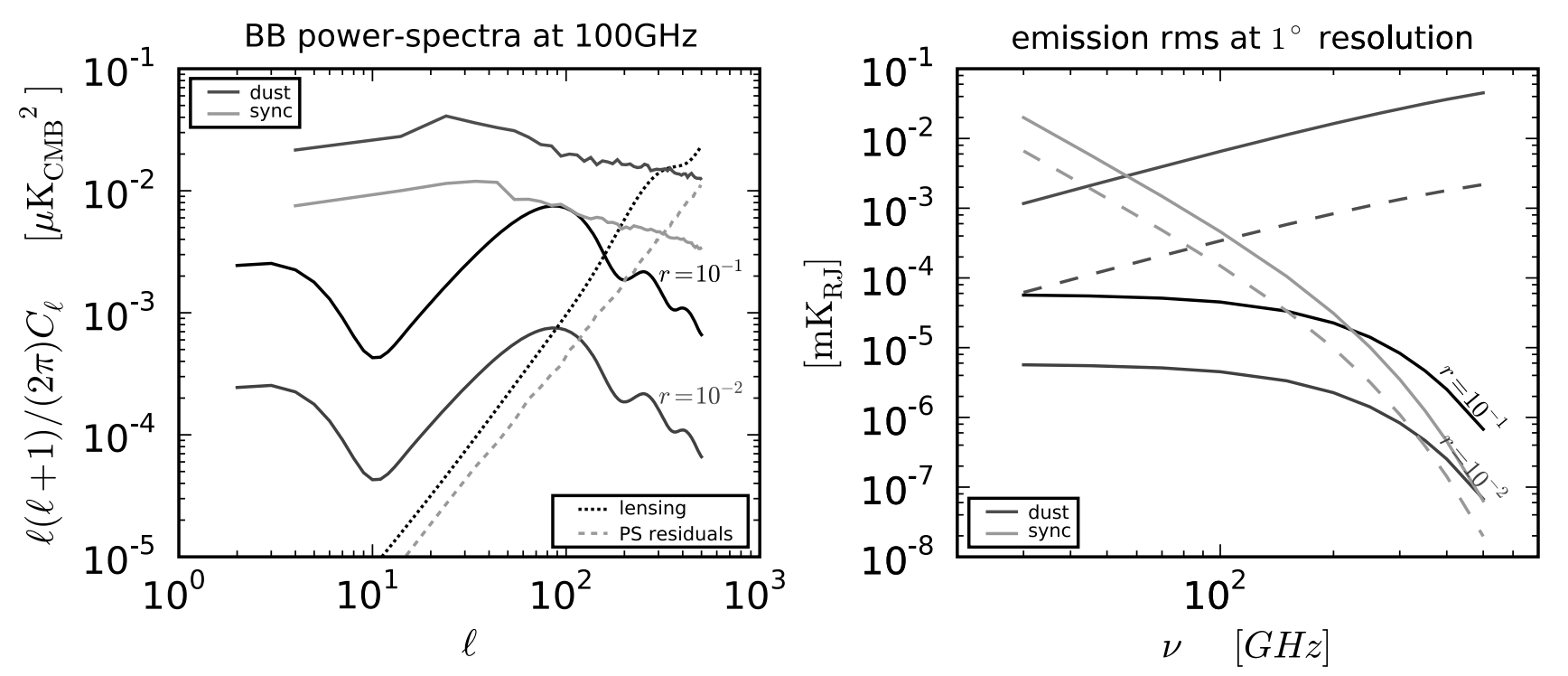

Fig. 1. Respective emission levels of the various components as predicted by the PSM. Left: predicted power spectra of the various components at $100 \mathrm{GHz}$, compared to $\mathrm{CMB}$ and lensing level for standard cosmology and various values of $r(\tau=0.07$, and other cosmological parameters follow Dunkley et al. 2008b). The power spectra of diffuse galactic foregrounds are computed using the cleanest 55\% of the polarised sky. The power spectrum from residual point sources is computed assuming that all sources brighter than 500 mJy (in temperature) in one of the Planck channels have been cut out. Right: typical frequency-dependence of the contributions to B-type polarisation of CMB, synchrotron and dust, at 1 degree resolution. The dashed lines correspond to the mean level of fluctuation as computed outside the mask used for the power spectra shown in the left panel.

this map with sky surveys at $1.4 \mathrm{GHz}$ (Reich \& Reich 1986) and $2.3 \mathrm{GHz}$ (Jonas et al. 1998), Giardino et al. (2002) and Platania et al. (2003) have derived nearly full sky spectral index maps. Using the measurement from WMAP, Bennett et al. (2003) derived the spectral index between $408 \mathrm{MHz}$ and $23 \mathrm{GHz}$. Compared to the former results, it showed a significant steepening toward $\beta_{s}=-3$ around $20 \mathrm{GHz}$, and a strong galactic plane feature with flatter spectral index. This feature was first interpreted as a flatter cosmic ray distribution in star forming regions. Recently, however, taking into account the presence, at $23 \mathrm{GHz}$, of additional contribution from a possible anomalous emission correlated with the dust column density, Miville-Deschênes et al. (2008) found no such pronounced galactic feature, in better agreement with lower frequency results. The spectral index map obtained in this way is consistent with $\beta_{s}=-3 \pm 0.06$. There is, hence, still significant uncertainty on the exact variability of the synchrotron spectral index, and in the amplitude of the steepening if any.

\subsubsection{Synchrotron polarisation}

If the electron density follows a power law of index $p$, the synchrotron polarisation fraction reads:

$f_{s}=3(p+1) /(3 p+7)$.

For $p=3$, we get $f_{s}=0.75$, a polarisation fraction which varies slowly for small variations of $p$. Consequently, the intrinsic synchrotron polarisation fraction should be close to constant on the sky. However, geometric depolarisation arises due to variations of the polarisation angle along the line of sight, partial cancellation of polarisation occurring for superposition of emission with orthogonal polarisation directions. Current measurements show variations of the observed polarisation value from about $10 \%$ near the galactic plane, to $30-50 \%$ at intermediate to high galactic latitudes (Macellari et al. 2008).

\subsubsection{Our model of synchrotron}

In summary, the B-mode intensity of the synchrotron emission is modulated by the density of cosmic rays, the slope of their spectra, the intensity of the magnetic field, its orientation, and the coherence of the orientation along the line of sight. This makes the amplitude and frequency scaling of the polarised synchrotron signal dependant on the sky position in a rather complex way.

For the purpose of the present work, we use the synchrotron model proposed in Miville-Deschênes et al. (2008) (model 4). It relies on the synchrotron polarised template at $23 \mathrm{GHz}$ measured by WMAP, and the computation of a spectral index map $\beta_{s}(\xi)$ used to extrapolate the template to other frequency following Eq. (1). This model also defines a pixel-dependent geometric depolarisation factor $g(\xi)$, computed as the ratio between the polarisation expected theoretically from Eq. (2), and the polarisation actually observed. This depolarisation, assumed to be due to varying orientations of the galactic magnetic field along the line of sight, is used also for modelling polarised dust emission (see below).

As an additional refinement, we also investigate the impact of a slightly modified frequency dependence with a running spectral index in Sect. 5. For this purpose, the synchrotron emission Stokes parameters $\left(S_{v}^{X}(\xi)\right.$ for $\left.X \in\{Q, U\}\right)$, at frequency $v$ and in direction $\xi$ on the sky, will be modelled instead as:

$S_{v}^{X}(\xi)=S_{v_{0}}^{X}(\xi)\left(\frac{v}{v_{0}}\right)^{\beta_{s}(\xi)+C(\xi) \log \left(v / v_{0}\right)}$

where $S_{v_{0}}^{X}(\xi)$ is the WMAP measurement at $v_{0}=23 \mathrm{GHz}, \beta_{s}$ the synchrotron spectral index map (Miville-Deschênes et al. 2008), 
and $C(\xi)$ a synthetic template of the curvature of the synchrotron spectral index.

The reconstructed B-modes map of the synchrotrondominated sky emission at $30 \mathrm{GHz}$ is shown in Fig. 2 (synthesis of $\mathrm{B}$-mode maps from $Q / U$ maps is described further along with the pipeline presentation in Sect. 4.1).

\subsection{Dust}

The thermal emission from heated dust grains is the dominant galactic signal at frequencies higher than $100 \mathrm{GHz}$ (Fig. 1). Polarisation of starlight by dust grains indicates partial alignment of elongated grains with the galactic magnetic field (see Lazarian (2007) for a review of possible alignment mechanisms). Partial alignment of grains should also result in polarisation of the far infrared dust emission.

Contributions from a wide range of grain sizes and compositions are required to explain the infrared spectrum of dust emission from 3 to $1000 \mu \mathrm{m}$ (Désert et al. 1990; Li \& Draine 2001). At long wavelengths of interest for CMB observations (above $100 \mu \mathrm{m}$ ), the emission from big grains, at equilibrium with the interstellar radiation field, should dominate.

\subsubsection{Dust thermal emission law}

There is no single theoretical emission law for dust, which is composed of many different populations of particles of matter. On average, an emission law can be fit to observational data. In the frequency range of interest for CMB observations, Finkbeiner et al. (1999) have shown that the dust emission in intensity is well modelled by emission from a two components mixture of silicate and carbon grains. For both components, the thermal emission spectrum is modelled as a modified grey-body emission, $D_{v} \sim B_{v}(T) v^{\alpha}$, with different emissivity spectral index $\alpha$ and different equilibrium temperature $T$.

\subsubsection{Dust polarisation}

So far, dust polarisation measurements have been mostly concentrated on specific regions of emission, with the exception of the Archeops balloon-borne experiment (Benoît et al. 2004), which has mapped the emission at $353 \mathrm{GHz}$ on a significant part of the sky, showing a polarisation fraction around 4-5\% and up to $10 \%$ in some clouds. This is in rough agreement with what could be expected from polarisation of starlight (Fosalba et al. 2002; Draine \& Fraisse 2008). Macellari et al. (2008) show that dust fractional polarisation in WMAP5 data depends on both frequency and latitude, but is typically about $3 \%$ and anyway below $7 \%$.

Draine \& Fraisse (2008) have shown that for particular mixtures of dust grains, the intrinsic polarisation of the dust emission could vary significantly with frequency in the $100-800 \mathrm{GHz}$ range. Geometrical depolarisation caused by integration along the line of sight also lowers the observed polarisation fraction.

\subsubsection{Our model of dust}

To summarise, dust produces polarised light depending on grains shape, size, composition, temperature and environment. The polarised light is then observed after integration along a line of sight. Hence, the observed polarisation fraction of dust depends on its three-dimensional distribution, and of the geometry of the galactic magnetic field. This produces a complex pattern which

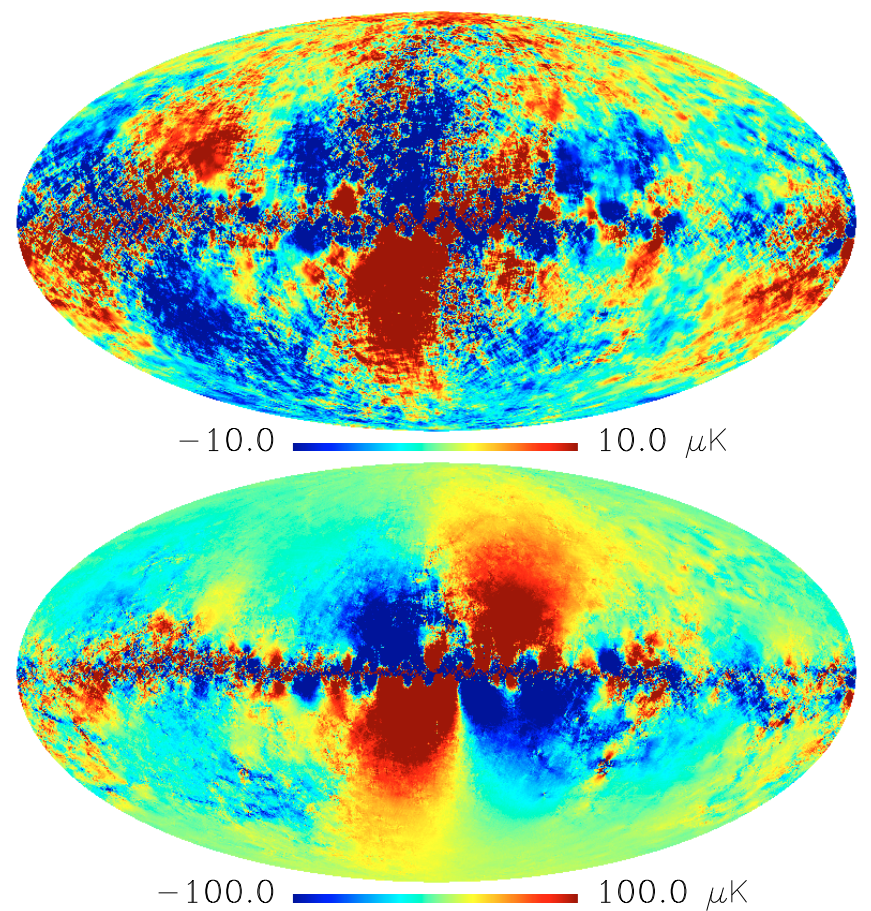

Fig. 2. B-modes of the galactic foreground maps (synchrotron + dust) as simulated using v1.6.4 of the PSM. Top: synchrotron-dominated emission at $30 \mathrm{GHz}$, Bottom: dust-dominated emission at $340 \mathrm{GHz}$.

is likely to be only partially coherent from one channel to another.

Making use of the available data, the PSM models polarised thermal dust emission by extrapolating dust intensity to polarisation intensity assuming an intrinsic polarisation fraction $f_{d}$ constant across frequencies. This value is set to $f_{d}=0.12$ to be consistent with maximum values observed by Archeops (Benoît et al. 2004) and is in good agreement with the WMAP $94 \mathrm{GHz}$ measurement. The dust intensity $\left(D_{v}^{T}\right)$, traced by the template map at $100 \mu \mathrm{m}$ from Schlegel et al. (1998), is extrapolated using Finkbeiner et al. (1999, model \#7) to frequencies of interest. The stokes $Q$ and $U$ parameters (respectively $D^{Q}$ and $D^{U}$ ) are then obtained as:

$D_{v}^{Q}(\xi)=f_{d} g(\xi) D_{v}^{T}(\xi) \cos (2 \gamma(\xi))$
$D_{v}^{U}(\xi)=f_{d} g(\xi) D_{v}^{T}(\xi) \sin (2 \gamma(\xi))$

The geometric "depolarisation" factor $g$ is a modified version of the synchrotron depolarisation factor (computed from WMAP measurements). Modifications account for differences of spatial distribution between dust grains and energetic electrons, and are computed using the magnetic field model presented in MivilleDeschênes et al. (2008). The polarisation angle $\gamma$ is obtained from the magnetic field model on large scales and from synchrotron measurements in WMAP on scales smaller than 5 degrees. The maps $\gamma(\xi)$ and $g(\xi)$ are shown in Fig. 3. Figure 2 shows the B-modes of dust at $340 \mathrm{GHz}$ using this model. In spite of the fact that the direction of polarisation of dust and synchrotron processes is determined by the same galactic magnetic field, differences in the 3-D distributions and in the depolarisation factors result in quite different B-mode polarisation patterns.

\subsubsection{Anomalous dust}

If the anomalous dust emission, which may account for a significant part of the intensity emission in the range $10-30 \mathrm{GHz}$ 

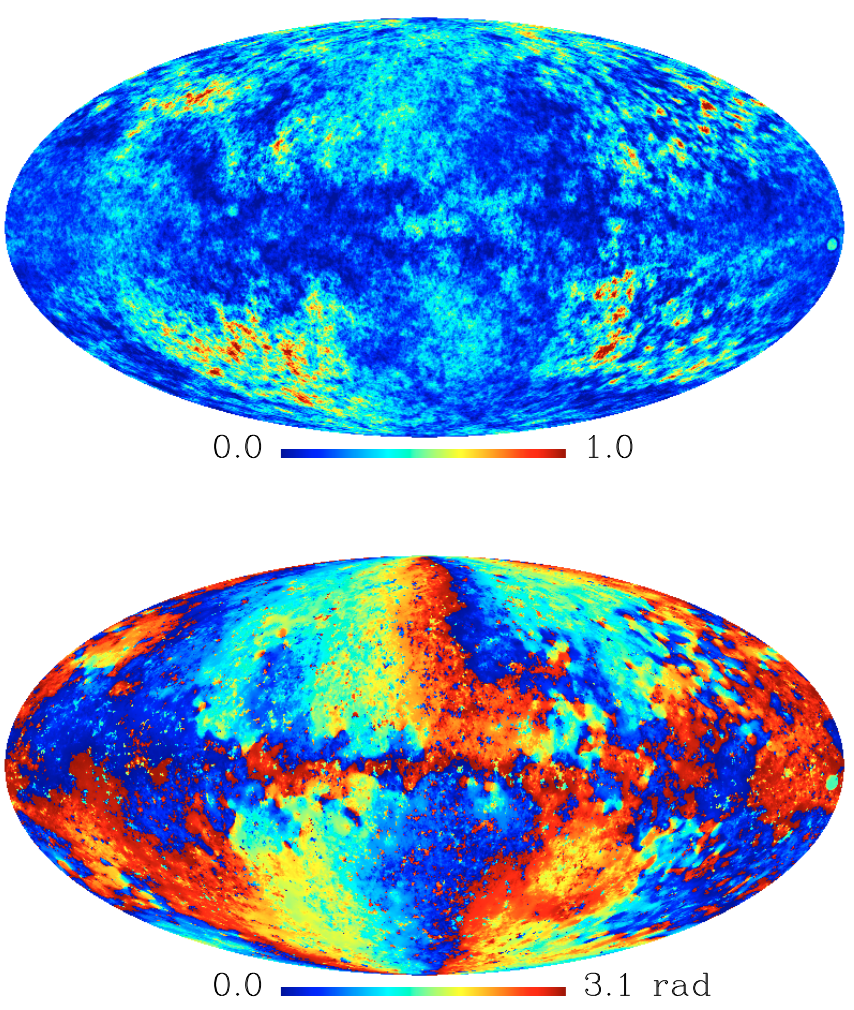

Fig. 3. Maps of the depolarisation factor $g$ (upper panel) and polarisation angle $\gamma$ (lower panel) entering in the model of dust polarised emission.

(Finkbeiner 2004; de Oliveira-Costa et al. 2004; MivilleDeschênes et al. 2008), can be interpreted as spinning dust grains emission (Draine \& Lazarian 1998), it should be slightly polarised under $35 \mathrm{GHz}$ (Battistelli et al. 2006), and only marginally polarised at higher frequencies (Lazarian \& Finkbeiner 2003). For this reason, it is neglected (and not modelled) here. However, we should keep in mind that there exist other possible emission processes for dust, like the magnetodipole mechanism, which can produce highly polarised radiation, and could thus contribute significantly to dust polarisation at low frequencies, even if subdominant in intensity (Lazarian \& Finkbeiner 2003).

\subsection{Other processes}

The left panel in Fig. 1 presents the respective contribution from the various foregrounds as predicted by the PSM at $100 \mathrm{GHz}$. Synchrotron and dust polarised emission, being by far the strongest contaminants on large scales, are expected to be the main foregrounds for the measurement of primordial B-modes. In this work, we thus mainly focus on the separation from these two diffuse contaminants. However, other processes yielding polarised signals at levels comparable with either the signal of interest, or with the sensitivity of the instrument used for B-mode observation, have to be taken into account.

\subsubsection{Free-free}

Free-free emission is assumed unpolarised to first order (the emission process is not intrinsically linearly polarised), even if, in principle, low level polarisation by Compton scattering could exist at the edge of dense ionised regions. In WMAP data analysis, Macellari et al. (2008) find an upper limit of $1 \%$ for free-free polarisation. At this level, free-free would have to be taken into account for measuring CMB B-modes for low values of $r$. As this is just an upper limit however, no polarised free-free is considered for the present work.

\subsubsection{Extra-galactic sources}

Polarised emission from extra-galactic sources is expected to be faint below the degree scale. Tucci et al. (2005), however, estimate that radio sources become the major contaminant after subtraction of the galactic foregrounds. It is, hence, an important foreground at high galactic latitudes. In addition, the point source contribution involves a wide range of emission processes and superposition of emissions from several sources, which makes this foreground poorly coherent across frequencies, and hence difficult to subtract using methods relying on the extrapolation of template emission maps.

The PSM provides estimates of the point source polarised emission. Source counts are in agreement with the prediction of de Zotti et al. (2005), and with WMAP data. For radio-sources, the degree of polarisation for each source is randomly drawn from the observed distribution at $20 \mathrm{GHz}$ (Ricci et al. 2004). For infrared sources, a distribution with mean polarisation degree of 0.01 is assumed. For both populations, polarisation angles are uniformly drawn in $[0-2 \pi$ [. The emission of a number of known galactic point sources is also included in PSM simulations.

\subsubsection{Lensing}

The last main contaminant to the primordial B-mode signal is lensing-induced B-type polarisation, the level of which should be of the same order as that of point sources (left panel of Fig. 1). For the present work, no sophisticated lensing cleaning method is used. Lensing effects are modelled and taken into account only at the power spectrum level and computed using the CAMB software package ${ }^{2}$, based itself on the CMBFAST software (Zaldarriaga et al. 1998; Zaldarriaga \& Seljak 2000).

\subsubsection{Polarised Sunyaev-Zel'dovich effect}

The polarised Sunyaev Zel'dovich effect (Sazonov \& Sunyaev 1999; Audit \& Simmons 1999; Seto \& Pierpaoli 2005), is expected to be very subdominant and is neglected here.

\subsection{Uncertainties on the foreground model}

Due to the relative lack of experimental constraints from observation at millimetre wavelengths, uncertainties on the foreground model are large. The situation will not drastically improve before the Planck mission provides new observations of polarised foregrounds. It is thus very important to evaluate, at least qualitatively, the impact of such uncertainties on component separation errors for B-mode measurements.

We may distinguish two types of uncertainties, which impact differently the separation of CMB from foregrounds. One concerns the level of foreground emission, the other its complexity. Quite reliable constraints on the emission level of polarised synchrotron at $23 \mathrm{GHz}$ are available with the WMAP

2 http://camb.info 
measurement, up to the few degrees scale. Extrapolation to other frequencies and smaller angular scales may be somewhat insecure, but uncertainties take place where this emission becomes weak and subdominant. The situation is worse for the polarised dust emission, which is only weakly constrained from WMAP and Archeops at 94 and $353 \mathrm{GHz}$. The overall level of polarisation is constrained only in the galactic plane, and its angular spectrum is only roughly estimated. In addition, variations of the polarisation fraction (Draine \& Fraisse 2008) may introduce significant deviations to the frequency scaling of dust B-modes.

Several processes make the spectral indexes of dust and synchrotron vary both in space and frequency. Some of this complexity is included in our baseline model, but some aspects, like the dependence of the dust polarisation fraction with frequency and the steepening of the synchrotron spectral index, remain poorly known and are not modelled in our main set of simulations. In addition, uncharacterised emission processes have been neglected. This is the case for anomalous dust, or polarisation of the free-free emission through Compton scattering. If such additional processes for polarised emission exist, even at a low level, they would decrease the coherence of galactic foreground emission between frequency channels, and hence our ability to predict the emission in one channel knowing it in the others - a point of much importance for any component separation method based on the combination of multi-frequency observations.

The component separation as performed in this paper, hence, is obviously sensitive to these hypotheses. We will dedicate a part of the discussion to assess the impact of such modelling errors on our conclusions.

\section{Estimating $r$ with contaminants}

Let us now turn to a presentation of the component separation (and parameter estimation) method used to derive forecasts on the tensor to scalar ratio measurements. Note that in principle, the best analysis of CMB observations should simultaneously exploit measurements of all fields $(T, E$, and $B)$, as investigated already by Aumont \& Macías-Pérez (2007). Their work, however, addresses an idealised problem. For component separation of temperature and polarisation together, the best approach is likely to depend on the detailed properties of the foregrounds (in particular on any differences, even small, between foreground emissions laws in temperature and in polarisation) and of the instrument (in particular noise correlations, and instrumental systematics). None of this is available for the present study. For this reason, we perform component separation in B-mode maps only. Additional issues such as disentangling $E$ from $B$ in cases of partial sky coverage for instance, or in the presence of instrumental systematic effects, are not investigated here either. Relevant work can be found in Kaplan \& Delabrouille (2002); Challinor et al. (2003); Hu et al. (2003); Rosset et al. (2007); Smith \& Zaldarriaga (2007).

For low values of tensor fluctuations, the constraint on $r$ is expected to come primarily from the B-mode polarisation. B-modes indeed are not affected by the cosmic variance of the scalar perturbations, contrarily to E-modes and temperature anisotropies. In return, B-mode signal would be low and should bring little constraint on cosmological parameters other than $r$ (and, possibly, the tensor spectral index $n_{t}$, although this additional parameter is not considered here). Decoupling the estimation of $r$ (from B-modes only) from the estimation of other cosmological parameters (from temperature anisotropies, from E-modes, and from additional cosmological probes) thus becomes a reasonable hypothesis for small values of $r$. As we are primarily interested in accurate handling of the foreground emission, we will make the assumption that all cosmological parameters but $r$ are perfectly known. Further investigation of the coupling between cosmological parameters can be found in Colombo et al. (2008) and Verde et al. (2006), and this question is discussed a bit further in Sect. 5.4.

\subsection{Simplified approaches}

\subsubsection{Single noisy map}

The first obstacle driving the performance of an experiment being the instrumental noise, it is interesting to recall the limit on $r$ achievable in absence of foreground contamination in the observations. We thus consider first a single frequency observation of the CMB, contaminated by a noise term $n$ :

$x(\xi)=x^{\mathrm{cmb}}(\xi)+n(\xi)$

where $\xi$ denotes the direction in the sky. Assuming that $n$ is uncorrelated with the $\mathrm{CMB}$, the power spectra of the map reads:

$C_{\ell}=r \mathcal{S}_{\ell}+\mathcal{N}_{\ell}$

where $\mathcal{S}_{\ell}$ is the shape of the CMB power-spectrum (as set by other cosmological parameters), and $\mathcal{N}_{\ell}$ the power of the noise contamination. Neglecting mode to mode mixing effects from a mask (if any), or in general from incomplete sky coverage, and assuming that $n$ can be modelled as a Gaussian process, the log-likelihood function for the measured angular power spectrum reads:

$-2 \ln \mathcal{L}=\sum_{\ell}(2 \ell+1) f_{\text {sky }}\left[\ln \left(\frac{C_{\ell}}{\hat{C}_{\ell}}\right)+\frac{\hat{C}_{\ell}}{C_{\ell}}\right]+$ const.

The smallest achievable variance $\sigma_{r}^{2}$ in estimating $r$ is the inverse of the Fisher information $\mathcal{I}=-\boldsymbol{E}\left(\frac{\partial^{2} \ln \mathcal{L}}{\partial r^{2}}\right)$ which takes the form:

$\sigma_{r}^{-2}=\sum_{\ell=\ell_{\min }}^{\ell_{\max }} \frac{2 \ell+1}{2} f_{\mathrm{sky}}\left(\frac{\mathcal{S}_{\ell}}{r \mathcal{S}_{\ell}+\mathcal{N}_{\ell}}\right)^{2}$.

For a detector (or a set of detectors at the same frequency) of noise equivalent temperature $s$ (in $\mu \mathrm{K} \sqrt{s}$ ), and a mission duration of $t_{s}$ seconds, the detector noise power spectrum is $\mathcal{N}_{\ell}=\frac{4 \pi s^{2}}{B_{\ell}^{2} t_{s}} \mu \mathrm{K}^{2}$, with $B_{\ell}$ denoting the beam transfer function of the detector.

A similar approach to estimating $\sigma_{r}$ is used in Verde et al. (2006) where a single "cleaned" map is considered. This map is obtained by optimal combination of the detectors with respect to the noise and cleaned from foregrounds up to a certain level of residuals, which are accounted for as an extra Gaussian noise.

\subsubsection{Multi-map estimation}

Alternatively, we may consider observations in $F$ frequency bands, and form the $F \times 1$ vector of data $\boldsymbol{x}(\xi)$, assuming that each frequency is contaminated by $\boldsymbol{x}^{\text {cont }}$. This term includes all contaminations (foregrounds, noise, etc.). In the harmonic domain, denoting $\boldsymbol{A}_{\mathrm{cmb}}$ the emission law of the CMB (the unit vector when working in thermodynamic units):

$\boldsymbol{a}_{\ell m}=\boldsymbol{A}_{\mathrm{cmb}} a_{\ell m}^{\mathrm{cmb}}+\boldsymbol{a}_{\ell m}^{\mathrm{cont}}$ 
We then consider the $F \times F$ spectral covariance matrix $\mathrm{R}_{\ell}$ containing auto and cross-spectra. The CMB signal being uncorrelated with the contaminants, one has:

$\mathrm{R}_{\ell}=\mathrm{R}_{\ell}^{\mathrm{cmb}}+\mathrm{N}_{\ell}$

with the $\mathrm{CMB}$ contribution modelled as

$\mathrm{R}_{\ell}^{\mathrm{cmb}}(r)=r \mathcal{S}_{\ell} \boldsymbol{A}_{\mathrm{cmb}} \boldsymbol{A}_{\mathrm{cmb}}^{\dagger}$

and all contaminations contributing a term $\mathrm{N}_{\ell}$ to be discussed later. The dagger $(\dagger)$ denotes the conjugate transpose for complex vectors and matrices, and the transpose for real matrices (as $\boldsymbol{A}_{\mathrm{cmb}}$ ).

In the approximation that contaminants are Gaussian (and, here, stationary) but correlated, all the relevant information about the $\mathrm{CMB}$ is preserved by combining all the channels into a single filtered map. In the harmonic domain, the filtering operation reads:

$\tilde{a}_{\ell m}=\boldsymbol{W}_{\ell} \boldsymbol{a}_{\ell m}=a_{\ell m}^{\mathrm{cmb}}+\boldsymbol{W}_{\ell} \boldsymbol{a}_{\ell m}^{\mathrm{cont}}$

with

$\boldsymbol{W}_{\ell}=\frac{\boldsymbol{A}_{\mathrm{cmb}}^{\dagger} \mathrm{N}_{\ell}^{-1}}{\boldsymbol{A}_{\mathrm{cmb}}^{\dagger} \mathrm{N}_{\ell}^{-1} \boldsymbol{A}_{\mathrm{cmb}}}$.

We are back to the case of a single map contaminated by a characterised noise of spectrum:

$\mathcal{N}_{\ell}=E\left|\boldsymbol{W}_{\ell} \boldsymbol{a}_{\ell m}^{\text {cont }}\right|^{2}=\left(\boldsymbol{A}_{\mathrm{cmb}}^{\dagger} \mathrm{N}_{\ell}^{-1} \boldsymbol{A}_{\mathrm{cmb}}\right)^{-1}$.

If the residual $\boldsymbol{W}_{\ell} \boldsymbol{a}_{\ell m}^{\text {cont }}$ is modelled as Gaussian, the single-map likelihood (7) can be used.

The same filter is used by Amblard et al. (2007). Assuming that the foreground contribution is perfectly known, the contaminant terms $\mathrm{N}_{\ell}$ can be modelled as $\mathrm{N}_{\ell}=\mathrm{R}_{\ell}^{\text {noise }}+\mathrm{R}_{\ell}^{\mathrm{fg}}$. This approach thus permits to derive the actual level of contamination of the map in the presence of known foregrounds, i.e. assuming that the covariance matrix of the foregrounds is known.

\subsection{Estimating $r$ in the presence of unknown foregrounds with SMICA}

The two simplified approaches of Sects. 3.1.1 and 3.1.2 offer a way to estimate the impact of foregrounds in a given mission, by comparing the sensitivity on $r$ obtained in absence of foregrounds (from Eq. (8) when $\mathcal{N}_{\ell}$ contains instrumental noise only), and the sensitivity achievable with known foregrounds (when $\mathcal{N}_{\ell}$ contains the contribution of residual contaminants as well, as obtained from Eq. (13) assuming that the foreground correlation matrix is known).

A key issue, however, is that the solution and the error bar require the covariance matrix of foregrounds and noise to be known $^{3}$. Whereas the instrumental noise can be estimated accurately, assuming prior knowledge of the covariance of the foregrounds to the required precision is optimistic.

To deal with unknown foregrounds, we thus follow a different route which considers a multi-map likelihood (Delabrouille et al. 2003). If all processes are modelled as Gaussian isotropic, then standard computations yield:

$$
-2 \ln \mathcal{L}=\sum_{\ell}(2 \ell+1) f_{\text {sky }} K\left(\widehat{\mathrm{R}}_{\ell}, \mathrm{R}_{\ell}\right)+\text { cst. }
$$

3 The actual knowledge of the contaminant term is not strictly required to build the filter. It is required, however, to derive the contamination level of the filtered map. where $\widehat{\mathrm{R}}_{\ell}$ is the sample estimate of $\mathrm{R}_{\ell}$ :

$\widehat{\mathrm{R}}_{\ell}=\frac{1}{2 \ell+1} \frac{1}{f_{\mathrm{sky}}} \sum_{m=-\ell}^{\ell} \boldsymbol{a}_{l, m} \boldsymbol{a}_{l, m}^{\dagger}$

and where $K(\cdot, \cdot)$ is a measure of mismatch between two positive matrices given by:

$K(\widehat{\mathrm{R}}, \mathrm{R})=\frac{1}{2}\left[\operatorname{trace}\left(\mathrm{R}^{-1} \widehat{\mathrm{R}}\right)-\log \operatorname{det}\left(\mathrm{R}^{-1} \widehat{\mathrm{R}}\right)-F\right]$.

Expression (14) is nothing but the multi-map extension of (7).

If $\mathrm{N}_{\ell}$ is known and fixed, then the likelihood (Eq. (14)) depends only on the CMB angular spectrum and can be shown to be equal (up to a constant) to expression 7 with $C_{\ell}=r S_{\ell}$ and $\mathcal{N}_{\ell}$ given by Eq. (13). Thus this approach encompasses both the single map and filtered map approaches.

Unknown foreground contribution can be modelled as the mixed contribution of $D$ correlated sources:

$\mathrm{R}_{\ell}^{\mathrm{fg}}=\mathrm{A} \Sigma_{\ell} \mathrm{A}^{\dagger}$

where $\mathrm{A}$ is a $F \times D$ mixing matrix and $\Sigma_{\ell}$ is the $D \times D$ spectral covariance matrix of the sources. The model of the spectral covariance matrix of the observations is then:

$\mathrm{R}_{\ell}=r \mathcal{S}_{\ell} A_{\mathrm{cmb}} A_{\mathrm{cmb}}^{\dagger}+\mathrm{A} \Sigma_{\ell} \mathrm{A}^{\dagger}+\mathrm{R}_{\ell}^{\text {noise }}$.

We then maximise the likelihood (14) of the model with respect to $r, A$ and $\Sigma_{\ell}$.

We note that the foreground parameterisation in Eq. (17) is redundant, as an invertible matrix can be exchanged between $A$ and $\Sigma$, without modifying the actual value of $\mathrm{R}^{\mathrm{fg}}$. The physical meaning of this is that the various foregrounds are not identified and extracted individually, only their mixed contribution is characterised. If we are interested in disentangling the foregrounds as well, e.g. to separate synchrotron emission from dust emission, this degeneracy can be lifted by making use of prior information to constrain, for example, the mixing matrix. Our multi-dimensional model offers, however, greater flexibility. Its main advantage is that no assumption is made about the foreground physics. It is not specifically tailored to perfectly match the model used in the simulation. Because of this, it is generic enough to absorb variations in the properties of the foregrounds, as will be seen later-on, but specific enough to preserve identifiability in the separation of CMB from foreground emission. A more complete discussion of the SMICA method with flexible components can be found in Cardoso et al. (2008).

A couple last details on SMICA and its practical implementation are of interest here. For numerical purposes, we actually divide the whole $\ell$ range into $Q$ frequency bins $\mathcal{D}_{q}=$ $\left\{\ell_{q}^{\min }, \cdots, \ell_{q}^{\max }\right\}$, and form the binned versions of the empirical and true cross power-spectra:

$$
\begin{aligned}
& \widehat{\mathrm{R}}_{q}=\frac{1}{w_{q}} \sum_{\ell \in \mathcal{D}_{q}} \sum_{m=-\ell}^{\ell} \boldsymbol{a}_{l, m} \boldsymbol{a}_{l, m}^{\dagger} \\
& \mathrm{R}_{q}=\frac{1}{w_{q}} \sum_{\ell \in \mathcal{D}_{q}}(2 \ell+1) \mathrm{R}_{\ell}
\end{aligned}
$$

where $w_{q}$ is the number of modes in $\mathcal{D}_{q}$. It is appropriate to select the domains so that we can reasonably assume for each $\ell \in \mathcal{D}_{q}, \mathrm{R}_{\ell} \approx \mathrm{R}_{q}$. This means that spectral bins should be small enough to capture the variations of the power spectra. In practice results are not too sensitive to the choice of the spectral bin 
widths. Widths between 5 and 10 multipoles constitute a good tradeoff.

Finally, we compute the Fisher information matrix $\mathbf{I}_{i, j}(\boldsymbol{\theta})$ deriving from the maximised likelihood (14) for the parameter set $\boldsymbol{\theta}=\left(r, \mathrm{~A}, \Sigma_{1}, \cdots, \Sigma_{\mathrm{Q}}\right)$ :

$\mathrm{I}_{i, j}(\boldsymbol{\theta})=\frac{1}{2} \sum_{q} w_{q} \operatorname{trace}\left(\frac{\partial \mathbf{R}_{q}(\boldsymbol{\theta})}{\partial \theta_{i}} \mathbf{R}_{q}^{-1} \frac{\partial \mathbf{R}_{q}(\boldsymbol{\theta})}{\partial \theta_{j}} \mathbf{R}_{q}^{-1}\right)$.

The lowest achievable variance of the $r$ estimate is obtained as the entry of the inverse of the FIM corresponding to the parameter $r$ :

$\sigma_{r}^{2}=\mathrm{I}_{r, r}^{-1}$

\section{Predicted results for various experimental designs}

We now turn to the numerical investigation of the impact of galactic foregrounds on the measurements of $r$ with the following experimental designs:

- the PlancK space mission, due for launch early 2009, which, although not originally planned for B-mode physics, could provide a first detection if the tensor to scalar ratio $r$ is around 0.1 ;

- various versions of the EPIC space mission, either low cost and low resolution (EPIC-LC), or more ambitious versions (EPIC-CS and EPIC-2m);

- an ambitious (fictitious) ground-based experiment, based on the extrapolation of an existing design (the Clover experiment);

- an alternative space mission, with sensitivity performances similar to the EPIC-CS space mission, but mapping only a small (and clean) patch of the sky, and referred as the "deep field mission".

The characteristics of these instruments are summed-up in Table 1, and Fig. 4 illustrates their noise angular power spectra in polarisation

\subsection{Pipeline}

For each of these experiments, we set up one or more simulation and analysis pipelines, which include, for each of them, the following main steps:

- simulation of the sky emission for a given value of $r$ and a given foreground model, at the central frequencies and the resolution of the experiment;

- simulation of the experimental noise, assumed to be white, Gaussian and stationary;

- computation, for each of the resulting maps, of the coefficients of the spherical harmonic expansion of the B-modes $a_{\ell m}^{B}$;

- synthesis from those coefficients of maps of B-type signal only;

- for each experiment, a mask based on the B-modes level of the foregrounds is built to blank out the brightest features of the galactic emission (see Fig. 5). This mask is built with smooth edges to reduce mode-mixing in the pseudospectrum;

- statistics described in Eq. (18) are built from the masked B maps;

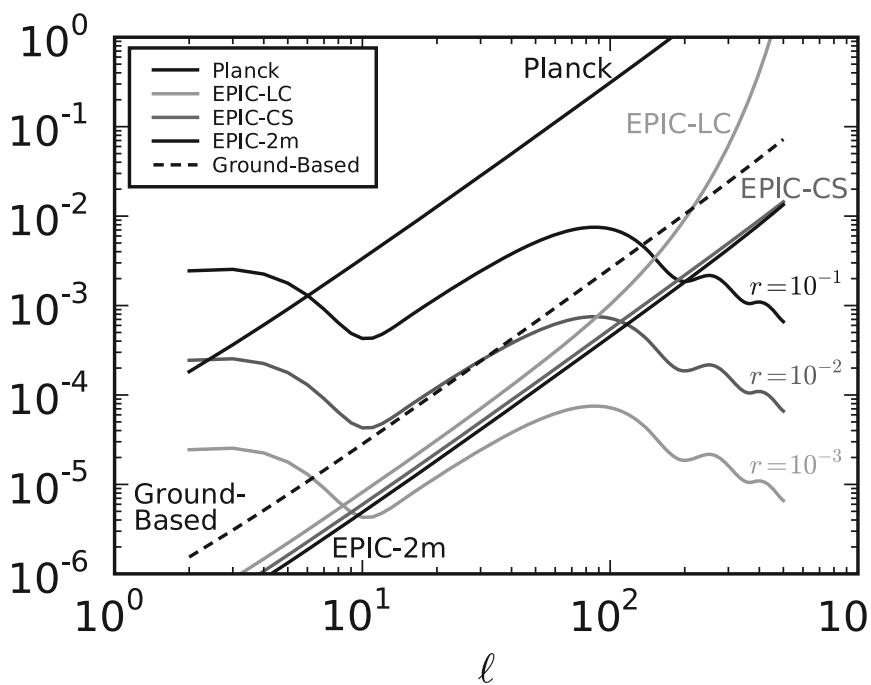

Fig. 4. Noise spectra of various experimental designs compared to Bmodes levels for $r=0.1,0.01$ and 0.001 . When computing the equivalent multipole noise level for an experiment, we assume that only the central frequency channels contribute to the CMB measurement and that external channels are dedicated to foreground characterisation.

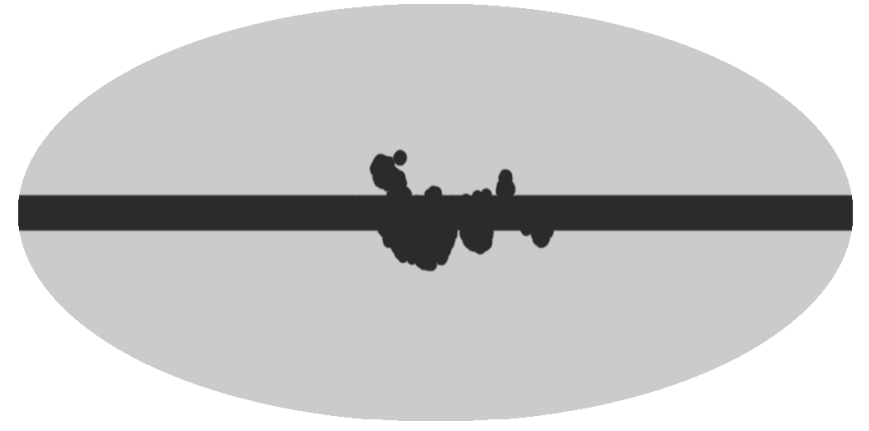

Fig. 5. Analysis mask for EPIC B maps, smoothed with a $1^{\circ}$ apodisation window.

- the free parameters of the model described in Sect. 3.2 are adjusted to fit these statistics. The shape of the CMB pseudospectrum that enters in the model, is computed using the mode-mixing matrix of the mask (Hivon et al. 2002);

- Error bars are derived from the Fisher information matrix of the model.

We insist that step 3 and 4 of this pipeline can be conducted as proposed only for full sky experiments. Experiments with partial coverage would have to deal with E/B mixing effect. Formally, step 5 (masking) could not come after step 2 and 3 in the processing of real-life part-sky data. As our main concern in this work is foreground contamination, we neglect this problem in the following, but one has to remain aware of this simplification when analysing results of Sect. 4.4. Some tuning of the pipeline is necessary for satisfactory foreground separation. The three main free parameters are the multipole range $\left[\ell_{\min }, \ell_{\max }\right]$, the dimension $D$ of the foreground component, and (for all-sky experiments) the size of the mask.

In practice we choose $\ell_{\min }$ according to the sky coverage and $\ell_{\max }$ according to the beam and the sensitivity. The value of $D$ is selected by iterative increments until the goodness of fit (as measured from the SMICA criterion on the data themselves, without knowledge of the input $\mathrm{CMB}$ and foregrounds) reaches its 
Table 1. Summary of experimental designs.

\begin{tabular}{lccccc}
\hline \hline Experiment & $\begin{array}{c}\text { Frequency } \\
(\mathrm{GHz})\end{array}$ & $\begin{array}{c}\text { Beam FWHM } \\
()^{\prime}\end{array}$ & $\begin{array}{c}\text { NET } \\
(\mu \mathrm{K} \sqrt{s})\end{array}$ & $\begin{array}{c}T_{\text {obs }} \\
(\mathrm{yr})\end{array}$ & $\begin{array}{c}\text { Sky coverage } \\
\left(f_{\text {sky }}\right)\end{array}$ \\
\hline PLANCK & $30,44,70$ & $33,24,14$ & $96,97,97$ & 1.2 & 1 \\
& $100,143,217,353$ & $10,7.1,5,5$ & $41,31,51,154$ & & \\
EPIC-LC & $30,40,60$ & $155,116,77$ & $28,9.6,5.3$ & 2 & 1 \\
& $90,135,200,300$ & $52,34,23,16$ & $2.3,2.2,2.3,3.8$ & & \\
EPIC-CS & $30,45,70,100$ & $15.5,10.3,6.6,4.6$ & $19,8,4.2,3.2$ & 4 & 1 \\
& $150,220,340,500$ & $3.1,2.1,1.4,0.9$ & $3.1,5.2,25,210$ & & \\
EPIC-2m & $30,45,70,100$ & $26,17,11,8$ & $18,7.6,3.9,3.0$ & 4 & 1 \\
Ground-Based & $150,320,340,500(, 800)$ & $5,3.5,2.3,1.5(, 0.9)$ & $2.8,4.4,20,180(, 28 \mathrm{k})$ & & \\
Deep field & $97,150,225$ & $7.5,5.5,5.5$ & $12,18,48$ & 0.8 & 0.01 \\
& $30,45,70,100$ & $15.5,10.3,6.6,4.6$ & $19,8,4.2,3.2$ & 4 & 0.01 \\
\hline
\end{tabular}

expectation. The mask is chosen in accordance to maximise the sky coverage for the picked value of $D$ (see Appendix A for further discussion of the procedure). The $f_{\text {sky }}=0.87$ mask in Fig. 5 , used for EPIC analysis, is based on a 10 degrees cut of the galactic plane. An additional cut of brightest region on the B-modes map is built by applying a threshold on the emission level at the central frequency of the instrument. The threshold can be adjusted to obtain the suitable sky coverage. These two masks are combined and edges are smoothed by a 1-degree transition window $^{4}$. For the Planck data analysis, the galactic plane cut is not necessary, and the emission level mask is used alone.

For each experimental design and fiducial value of $r$ we compute three kinds of error estimates which are recalled in Table 2:

- knowing the noise level and resolution of the instrument, we first derive from Eq. (8) the error $\sigma_{r}^{\text {noise-only }}$ set by the instrument sensitivity assuming no foreground contamination in the covered part of the sky. The global noise level of the instrument is given by $\mathcal{N}_{\ell}=\left(A_{\mathrm{cmb}}^{\dagger} \mathrm{N}_{\ell}^{-1} \boldsymbol{A}_{\mathrm{cmb}}\right)^{-1}$, where the only contribution to $\mathrm{N}_{\ell}$ comes from the instrumental noise: $\mathrm{N}_{\ell}=\mathrm{R}_{\ell}^{\text {noise }}=\operatorname{diag}\left(\frac{4 \pi s_{f}^{2}}{B_{\ell}, f^{2} t_{s}}\right)$;

- in the same way, we also compute the error $\sigma_{r}^{\text {known-foreground }}$ that would be obtained if foreground contribution $R^{\text {fg }}$ to the covariance of the observations was perfectly known, using $\mathrm{N}_{\ell}=\mathrm{R}_{\ell}^{\text {noise }}+\mathrm{R}_{\ell}^{\mathrm{fg}}$. Here we assume that $\mathrm{R}^{\mathrm{fg}}=\widehat{\mathrm{R}}^{\mathrm{fg}}$ where $\widehat{\mathrm{R}}^{\mathrm{fg}}$ is the sample estimate of $R^{\mathrm{fg}}$ computed from the simulated foreground maps;

- finally, we compute the error $\sigma_{r}^{\text {SMICA }}$ given by the Fisher information matrix of the model (Eq. (20)).

In each case, we also decompose the FIM in the contribution from large-scale modes $(\ell \leq 20)$ and the contribution from small scales $(\ell>20)$. We then compute error bars from these two separate contributions to give indications of the relative weight of the bump (due to reionisation) and the peak (at higher $\ell$ ) in the constraint of $r$.

We may notice that in some favourable cases (at low $\ell$, where the foregrounds dominate), the error estimate given by SMICA can be slightly more optimistic than the estimate obtained using the actual empirical value of the correlation matrix $\widehat{R}^{\mathrm{fg}}$. This reflects the fact that our modelling hypothesis, which imposes to $\mathrm{R}^{\mathrm{fg}}$ to be of rank smaller than $D$, is not perfectly verified in practice (see Appendix A for further discussion of this hypothesis). The (small) difference (an error on the estimation of $\sigma_{r}$ when

4 The mask apodisation code can be downloaded from http: //WWW . apc . univ-paris7. fr/ betoule/doku . php?id=software foregrounds are approximated by our model) has negligible impact on the conclusions of this work.

\subsection{Planck}

The Planck space mission will be the first all-sky experiment to give sensitive measurements of the polarised sky in seven bands between 30 and $353 \mathrm{GHz}$. The noise level of this experiment being somewhat too high for precise measurement of low values of $r$, we run our pipeline for $r=0.1$ and 0.3 . We predict a possible 3-sigma measurement for $r=0.1$ using SMICA (first lines in Table 2). A comparison of the errors obtained from SMICA, with the prediction in absence of foreground contamination, and with perfectly known foreground contribution, indicates that the error is dominated by cosmic variance and noise, foregrounds contributing to a degradation of the error of $\sim 30 \%$ and uncertainties on foregrounds for another increase around $30 \%$ (for $r=0.1$ ).

Figure 4 hints that a good strategy to detect primordial Bmodes with Planck consists in detecting the reionisation bump below $\ell=10$, which requires the largest possible sky coverage. Even at high latitude, a model using $D=2$ fails to fit the galactic emission, especially on large scales where the galactic signal is above the noise. Setting $D=3$, however, gives a satisfactory fit (as measured by the mismatch criterion) on 95 percent of the sky. It is therefore our choice for Planck.

We also note that a significant part of the information is coming from the reionisation bump $(\ell \leq 20)$. The relative importance of the bump increases for decreasing value of $r$, as a consequence of the cosmic variance reduction. For a signal-to-noise ratio corresponding roughly to the detection limit $(r=0.1)$, the stronger constraint is given by the bump (Appendix B gives further illustration of the relative contribution of each multipole). This has two direct consequences: the result is sensitive to the actual value of the reionisation optical depth and to reionisation history (as investigated by Colombo \& Pierpaoli 2008), and the actual capability of Planck to measure $r$ will depend on the level (and the knowledge of) instrumental systematics on large scales. Note that this numerical experiment estimates how well Planck can measure $r$ in the presence of foregrounds from B-modes only.

\subsection{EPIC}

We perform a similar analysis for three possible designs of the EPIC probe (Bock et al. 2008). EPIC-LC and EPIC-CS correspond respectively to the low cost and comprehensive solutions. EPIC-2m is an alternate design which contains one extra highfrequency channel (not considered in this study) dedicated to 
Table 2. Error prediction for various experimental designs, assumptions about foregrounds, and fiducial $r$ values.

\begin{tabular}{|c|c|c|c|c|c|c|c|c|c|c|c|c|c|c|}
\hline \multirow{2}{*}{ Case } & $r$ & $\begin{array}{r}\mathrm{N} \\
\sigma_{r} / r \\
\end{array}$ & $\begin{array}{l}\text { oise }-\mathrm{o} \\
\sigma_{r}^{\ell \leq 20} / r \\
\end{array}$ & $\sigma_{r}^{\ell>20} / r$ & \multicolumn{3}{|c|}{$\begin{array}{l}\text { Known foregrounds } \\
\sigma_{r} / r \quad \sigma_{r}^{\ell \leq 20} / r \sigma_{r}^{\ell \ell 20} / r\end{array}$} & \multicolumn{4}{|c|}{ SMICA $^{c}$} & $l_{\min }-l_{\max }$ & $f_{\text {sky }}$ & $D^{e}$ \\
\hline & 0.3 & 0.075 & 0.17 & 0.084 & 0.1 & 0.2 & 0.12 & 0.15 & 0.22 & 0.2 & 0.26 & & & \\
\hline & 0.1 & 0.17 & 0.25 & 0.22 & 0.23 & 0.34 & 0.32 & 0.29 & 0.34 & 0.55 & 0.086 & & 0.95 & 3 \\
\hline \multirow{2}{*}{ EPIC-LC } & 0.01 & 0.019 & 0.084 & 0.019 & 0.05 & 0.18 & 0.053 & 0.079 & 0.18 & 0.1 & 0.0098 & \multirow{2}{*}{$2-130$} & \multirow{2}{*}{0.86} & \\
\hline & 0.001 & 0.059 & 0.15 & 0.064 & 0.27 & 0.4 & 0.38 & 0.37 & 0.43 & 0.82 & 0.00088 & & & 4 \\
\hline \multirow{2}{*}{ EPIC-2m } & 0.01 & 0.016 & 0.083 & 0.016 & 0.027 & 0.12 & 0.027 & 0.032 & 0.11 & 0.036 & 0.0096 & \multirow{2}{*}{$2-300$} & \multirow{2}{*}{0.87} & 4 \\
\hline & 0.001 & 0.051 & 0.14 & 0.055 & 0.14 & 0.25 & 0.16 & 0.16 & 0.24 & 0.24 & 0.001 & & & \\
\hline \multirow{2}{*}{ EPIC-CS } & 0.01 & 0.017 & 0.084 & 0.017 & 0.029 & 0.12 & 0.03 & 0.036 & 0.11 & 0.041 & 0.0096 & \multirow{2}{*}{$2-300$} & \multirow{2}{*}{0.87} & 4 \\
\hline & 0.001 & 0.058 & 0.15 & 0.063 & 0.15 & 0.27 & 0.19 & 0.18 & 0.26 & 0.29 & 0.00098 & & & 4 \\
\hline \multirow{2}{*}{ Ground-based } & 0.1 & 0.083 & - & - & 0.15 & - & - & 0.24 & - & - & 0.11 & \multirow{2}{*}{$50-300$} & \multirow{2}{*}{0.01} & \\
\hline & 0.01 & 0.18 & - & - & 0.8 & - & - & 1.6 & - & - & 0.018 & & & 2 \\
\hline Grnd - base & 0.01 & 0.18 & - & - & 0.51 & - & - & 0.69 & - & - & 0.0065 & $50-300$ & 0.01 & 2 \\
\hline Deep field mission & 0.001 & 0.082 & - & - & 0.1 & - & - & 0.13 & - & - & 0.00092 & $50-300$ & 0.01 & 4 \\
\hline
\end{tabular}

${ }^{a}$ Error bars derived assuming no foreground contamination i.e. using $\mathrm{N}_{\ell}=\mathrm{R}^{\text {noise }}$ (see Eq. (8)). ${ }^{b}$ Same as ${ }^{a}$ but assuming perfectly known foreground contamination i.e. using $\mathrm{N}_{\ell}=\mathrm{R}^{\text {noise }}+\mathrm{R}^{\mathrm{fg}}$. ${ }^{c}$ Error bars obtained by the inversion of the FIM computed from the SMICA model at the point of convergence of the algorithm (see Eq. (20)). ${ }^{d}$ Estimated value at the convergence point in SMICA; detections at more than $4 \sigma$ are bold-faced. ${ }^{e}$ Size of the galactic component used in the model of SMICA.

additional scientific purposes besides CMB polarisation. We consider two values of $r, 0.01$ and 0.001 . For all these three experiments, the analysis requires $D=4$ for a reasonable fit, which is obtained using about $87 \%$ of the sky.

The two high resolution experiments provide measurements of $r=10^{-3}$ with a precision better than five sigma. For the lower values of $r$, the error is dominated by foregrounds and their presence degrades the sensitivity by a factor of 3 , as witnessed by the difference between $\sigma_{r}^{\text {noise-only }}$ and $\sigma_{r}^{\text {smica }}$. However, while the difference between the noise-only and the SMICA result is a factor 4-6 for EPIC-LC, it is only a factor about 2-3 for EPIC-CS and EPIC-2m. Increased instrumental performance (in terms of frequency channels and resolution) thus also allows for better subtraction of foreground contamination.

For all experiments considered, the constraining power moves from small scales to larger scale when $r$ decreases down to the detection limit of the instrument. In all cases, no information for the CMB is coming from $\ell>150$. Higher multipoles, however, are still giving constraints on the foreground parameters, effectively improving the component separation also on large scales.

\subsection{Small area experiments}

\subsubsection{Ground-based}

A different observation strategy for the measurement of B-modes is adopted for ground-based experiments that cannot benefit from the frequency and sky coverage of a space mission. Such experiments target the detection of the first peak around $\ell=100$, by observing a small but clean area (typically 1000 squaredegrees) in few frequency bands ( 2 or 3 ).

The test case we propose here is inspired from the announced performances of C $\ell$ over (North et al. 2008). The selected sky coverage is a 10 degree radius area centred on lon $=351^{\circ}$, lat $=-56^{\circ}$ in galactic coordinates. The region has been retained by the $\mathrm{C} \ell$ over team as a tradeoff between several issues including, in particular, foreground and atmospheric contamination. According to our polarised galactic foreground model, this also correspond to a reasonably clean part of the sky (within $30 \%$ of the cleanest).
The most interesting conclusion is that for $r=0.01$, although the raw instrumental sensitivity (neglecting issues like E-B mixing due to partial sky coverage) would allow a more than five sigma detection, galactic foregrounds cannot be satisfactorily removed with the scheme adopted here.

An interesting option would be to complement the measurement obtained from the ground, with additional data such as that of Planck, and extract $r$ in a joint analysis of the two data sets. To simply test this possibility here, we complement the ground data set with a simulation of the Planck measurements on the same area. This is equivalent to extend the frequency range of the ground experiment with less sensitive channels. We find a significant improvement of the error-bar from $1.6 \times 10^{-2}$ to $0.69 \times 10^{-2}$, showing that a joint analysis can lead to improved component separation. The degradation of sensitivity due to foreground remains however higher than for a fully sensitive space mission (as witnessed by the following section). This last result is slightly pessimistic as we do not make use of the full Planck data set but use it only to constrain foregrounds in the small patch. However considering the ratio of sensitivity between the two experiments, it is likely that there is little to gain by pushing the joint analysis further.

\subsubsection{Deep field space mission}

We may also question the usefulness of a full-sky observation strategy for space-missions, and consider the possibility to spend the whole observation time mapping deeper a small but clean region. We investigate this alternative using an hypothetical experiment sharing the sensitivity and frequency coverage of the EPIC-CS design, and the sky coverage of the ground-based experiment. Although the absence of strong foreground emission may permit a design with a reduced frequency coverage, we keep a design similar to EPIC-CS to allow comparisons. In addition, the relative failure of the ground-based design to disentangle foregrounds indicates that the frequency coverage cannot be freely cut even when looking in the cleanest part of the sky. In the same way, to allow straightforward comparison with the ground-based case we stick to the same sky coverage, although in principle, without atmospheric constraints, slightly better sky areas could be selected. 
In spite of the increased cosmic variance due to the small sky coverage, the smaller foreground contribution allows our harmonic-based foreground separation with SMICA to achieve better results with the "deep field" mission than with the full sky experiment, when considering only diffuse galactic foreground. However, this conclusion does not hold if lensing is considered as will be seen in the following section.

We may also notice that, despite the lower level of foregrounds, the higher precision of the measurement requires the same model complexity $(D=4)$ as for the full sky experiment to obtain a good fit. We also recall that our processing pipeline does not exploit the spatial variation of foreground intensity, and is, in this sense, suboptimal, in particular for all-sky experiments. Thus, the results presented for the full-sky experiment are bound to be slightly pessimistic which tempers further the results of this comparison between deep field and full sky mission. This is further discussed below. Finally, note that here we also neglect issues related to partial sky coverage that would be unavoidable in this scheme.

\subsection{Comparisons}

\subsubsection{Impact of foregrounds: the ideal case}

As a first step, the impact of foregrounds on the capability to measure $r$ with a given experiment, if foreground covariances are known, is a measure of the adequacy of the experiment to deal with foreground contamination. Figures for this comparison are computed using Eqs. (8) and (13), and are given in Table 2 (first two sets of three columns).

The comparison shows that for some experiments, $\sigma_{r} / r$ in the "noise-only" and the "known foregrounds" cases are very close. This is the case for Planck and for the deep field mission. For these experiments, if the second order statistics of the foregrounds are known, galactic emission does not impact much the measurement. For other experiments, the "known foregrounds" case is considerably worse than the "noise-only" case. This happens, in particular, for a ground based experiment when $r=0.01$, and for EPIC-LC.

If foreground contamination was Gaussian and stationary, and in absence of priors on the CMB power spectrum, the linear filter of Eq. (12) would be the optimal filter for CMB reconstruction. The difference between $\sigma_{r}$ in the "noise-only" and the "known foregrounds" cases would be a good measure of how much the foregrounds hinder the measurement of $r$ with the experiment considered. A large difference would indicate that the experimental design (number of frequency channels and sensitivity in each of them) is inadequate for "component separation".

However, since foregrounds are neither Gaussian nor stationary, the linear filter of Eq. (12) is not optimal. Even if we restrict ourselves to linear solutions, the linear weights given to the various channels should obviously depend on the local properties of the foregrounds. Hence, nothing guarantees that we can not deal better with the foregrounds than using a linear filter in harmonic space. Assuming that the covariance matrix of the foregrounds is known, the error in Eq. (8) with $\mathcal{N}_{\ell}$ from Eq. (13) is a pessimistic bound on the error on $r$. The only conclusion that can be drawn is that the experiment does not allow effective component separation with the implementation of a linear filter in harmonic space. There is, however, no guarantee either that an other approach to component separation would yield better results. Hence, the comparison of the noise-only and known foregrounds cases shown here gives an upper limit of the impact of foregrounds, if they were known.

\subsubsection{Effectiveness of the blind approach}

Even if in some cases the linear filter of Eq. (12) may not be fully optimal, it is for each mode $\ell$ the best linear combination of observations in a set of frequency channels, to reject maximally contamination from foregrounds and noise, and minimise the error on $r$. Other popular methods as decorrelation in direct space, such as the so-called "internal linear combination", and other linear combinations cannot do better, unless they are implemented locally in both pixel and harmonic space simultaneously, using for instance spherical needlets as in Delabrouille et al. (2008). Such localisation is not considered in the present work.

Given this, the next question that arises is how well the spectral covariance of the foreground contamination can be actually constrained from the data, and how this uncertainty impact the measurement of $r$. The answer to this question is obtained by comparing the second and third sets of columns of Table 2.

In all cases, the difference between the results obtained assuming perfect knowledge of the foreground residuals, and those obtained after the blind estimation of the foreground covariances with SMICA, are within a factor of 2. For EPIC-2m and the deep field mission, the difference between the two is small, which means that SMICA allows for component separation very effectively. For a ground based experiment with three frequency channels, the difference is very significant, which means that the data does not allow a good blind component separation with SMICA.

Comparing column set 1 (noise-only) and 3 (blind approach with SMICA) gives the overall impact of unknown galactic foregrounds on the measurement of $r$ from B-modes with the various instruments considered. For Planck, EPIC-2m, or a deep field mission with 8 frequency channels, the final error bar on $r$ is within a factor of 2 of what would be achievable without foregrounds. For EPIC-LC, or even worse for a ground-based experiment, foregrounds are likely to impact the outcome of the experiment quite significantly. For this reason, EPIC-2m and the deep field mission seem to offer better perspectives for measuring $r$ in the presence of foregrounds.

\subsubsection{Full sky or deep field}

The numerical investigations performed here allow -to some extent- to compare what can be achieved with our approach in two cases of sky observation strategies with the same instrument. For EPIC-CS, it has been assumed that the integration time is evenly spread on the entire sky, and that $87 \%$ of the sky is used to measure $r$. For the "deep field" mission, $1 \%$ of the sky only is observed with the same instrument, with much better sensitivity per pixel (by a factor of 10).

Comparing $\sigma_{r} / r$ between the two in the noise-only case shows that the full sky mission should perform better (by a factor 1.4) if the impact of the foregrounds could be made to be negligible. This is to be expected, as the cosmic or "sample" variance of the measurement is smaller for larger sky coverage. After component separation however, the comparison is in favour of the deep field mission, which seems to perform better by a factor 1.4 also. The present work, however, does not permit to conclude on what is the best strategy for two reasons. First, this study concentrates on the impact of diffuse galactic foregrounds which are not expected to be the limiting issue of the deep field design. And secondly, in the case of a deep field, the properties of the (simulated) foreground emission are more homogeneous in the observed area, and thus the harmonic filter of Eq. (12) is close to optimal everywhere. For the full sky mission, however, the filter is obtained as a compromise minimising the overall 
error $\ell$ by $\ell$, which is not likely to be the best everywhere on the sky. Further work on component separation, making use of a localised version of SMICA, is needed to conclude on this issue. A preliminary version of SMICA in wavelet space is described in Moudden et al. (2004), but applications to CMB polarisation and full sky observations require specific developments.

\section{Discussion}

The results presented in the previous section have been obtained using a number of simplifying assumptions. First of all, only galactic foregrounds (synchrotron and dust) are considered. It has been assumed that other foregrounds (point sources, lensing) can be dealt with independently, and thus will not impact much the overall results. Second, it is quite clear that the results may depend on details of the galactic emission, which might be more complex than what has been used in our simulations. Third, most of our conclusions depend on the accuracy of the determination of the error bars from the Fisher information matrix. This method, however, only provides an approximation, strictly valid only in the case of Gaussian processes and noise. Finally, the measurement of $r$ as performed here assumes a perfect prediction (from other sources of information) of the shape of the BB spectrum. In this section, we discuss and quantify the impact of these assumptions, in order to assess the robustness of our conclusions.

\subsection{Small-scale contamination}

\subsubsection{Impact of lensing}

Limitations on tensor mode detection due to lensing have been widely investigated in the literature, and cleaning methods, based on the reconstruction of the lensed B-modes from estimation of the lens potential and unlensed CMB E-modes, have been proposed (Knox \& Song 2002; Hirata \& Seljak 2003; Kesden et al. 2003; Lewis \& Challinor 2006). However, limits on $r$ achievable after such "delensing" (if any) are typically significantly lower than limits derived in Sect. 4, for which foregrounds and noise dominate the error.

In order to check whether the presence of lensing can significantly alter the detection limit, we proceed as follows: assuming no specific reconstruction of the lens potential, we include lensing effects in the simulation of the CMB (at the power spectrum level). The impact of this on the second order statistics of the $\mathrm{CMB}$ is an additional contribution to the CMB power spectrum. This extra term is taken into account on the CMB model used in SMICA. For this, we de-bias the CMB SMICA component from the (expectation value of) the lensing contribution to the power-spectrum. The cosmic variance of the lensed modes thus contributes as an extra "noise" which lowers the sensitivity to the primordial signal, and reduces the range of multipoles contributing significantly to the measurement. We run this lensing test case for the EPIC-CS and deep field mission. Table 3 shows a comparison of the constraints obtained with and without lensing in the simulation for a fiducial value of $r=0.001$. On large scales for EPIC-CS, lensing has negligible impact on the measurement of $r$ (the difference between the two cases is not significant on one single run of the component separation). On small scales, the difference becomes significant. Overall, $\sigma_{r} / r$ changes from 0.18 to 0.21 , which is not a very significant degradation of the measurement: lensing produces a $15 \%$ increase in the overall error estimate, the small-scale error (for $\ell>20$ ) being most impacted. For the small coverage mission, however, the
Table 3. Comparison of the constraints on $r$ with and without lensing (here $r=0.001$ ).

\begin{tabular}{lcccccc}
\hline \hline & \multicolumn{3}{c}{ Nolensing } & \multicolumn{3}{c}{ Lensing } \\
Experiment & $\sigma_{r} / r$ & $\sigma_{r}^{\ell \leq 20} / r$ & $\sigma_{r}^{\ell>20} / r$ & $\sigma_{r} / r$ & $\sigma_{r}^{\ell \leq 20} / r$ & $\sigma_{r}^{\ell>20} / r$ \\
\hline EPIC-CS & 0.18 & 0.26 & 0.29 & 0.21 & 0.26 & 0.38 \\
Deepfield & 0.13 & - & - & 1.1 & - & - \\
\hline
\end{tabular}

large cosmic variance of the lensing modes considerably hinder the detection.

Thus, at this level of $r$, if the reionisation bump is satisfactorily measured, the difference is perceptible but not very significant. Hence, lensing is not the major source of error for a full-sky experiment measuring $r$. It becomes however a potential problem for a small coverage experiment targeting the measurement of the recombination bump. Such a strategy would thus require efficient "delensing". Indications that "delensing" can be performed even in the presence of foregrounds in the case of a low noise and high resolution experiment can be found in Smith et al. (2008). However, a complete investigation of this case, accounting for all the complexity (diffuse foregrounds, point sources, lensing, modes-mixing effects), would be needed to conclude on the validity of a deep-field strategy.

\subsubsection{Impact of extra-galactic sources}

Although largely subdominant on scales larger than 1 degree, extra-galactic sources, in particular radio-sources, are expected to be the worst contaminant on small scales (see e.g. Tucci et al. 2004; Pierpaoli \& Perna 2004). Obviously, the strongest point sources are known, or (for most of them) will be detected by Planck. Their polarisation can be measured either by the B-mode experiment itself, or by dedicated follow-up. We make the assumption that point sources brighter than $500 \mathrm{mJy}$ in temperature (around 6000 sources) are detected, and that their polarised emission is subtracted from the polarisation observations. We stress that $500 \mathrm{mJy}$ is a conservative assumption as Planck is expected to have better detection thresholds.

The present level of knowledge about point sources does not allow a very accurate modelling of the contribution to the power spectra of the remaining point sources (those not subtracted by the $500 \mathrm{mJy}$ cut). For this reason we investigate their impact in two extreme cases: perfect modelling of their contribution to the power-spectra ("ideal" case), and no specific modelling at all ("no-model" case). Results of a SMICA run for both assumptions are compared to what is obtained in total absence of point sources ("no-ps" case), and are summarised in Table 4.

The bottom line of this investigation is that modelling properly the point sources statistical contribution is necessary to measure $r=0.001$. An insufficient model results in a biased estimator: for EPIC-CS the estimated $r$ is two times larger than expected, with a difference incompatible with the error bar, in spite of an increased standard deviation $\left(\sigma_{r}\right.$ increased by $+30 \%$ for $r=0.001$ ). An ideal model restores the goodness of fit of the no-ps case and suppresses the bias of the estimator. Still, the presence of point sources increases the variance of the measurement of $r$. In our experiment, the effect is not truly significant $\left(\sigma_{r}\right.$ shifting from 1.84 to $\left.1.91 \times 10^{-4}\right)$.

Figure 6 shows the mismatch criterion (from Eq. (16), using covariance matrixes binned in $\ell$ ) in the three cases. When no specific model of the point source contribution is used, some of their emission is nonetheless absorbed by the SMICA "galactic" com- 
Table 4. EPIC-CS measurement for three point sources cases.

\begin{tabular}{ccccccc}
\hline \hline$r$ & $r^{\text {no-ps }}$ & $r^{\text {ideal }}$ & $r^{\text {no-model }}$ & $\sigma_{r}^{\text {no-ps }}$ & $\sigma_{r}^{\text {ideal }}$ & $\sigma_{r}^{\text {no-model }}$ \\
\hline 0.001 & $1.07 \times 10^{-3}$ & $1.04 \times 10^{-3}$ & $2.00 \times 10^{-3}$ & $1.84 \times 10^{-4}$ & $1.91 \times 10^{-4}$ & $2.49 \times 10^{-4}$ \\
\hline
\end{tabular}

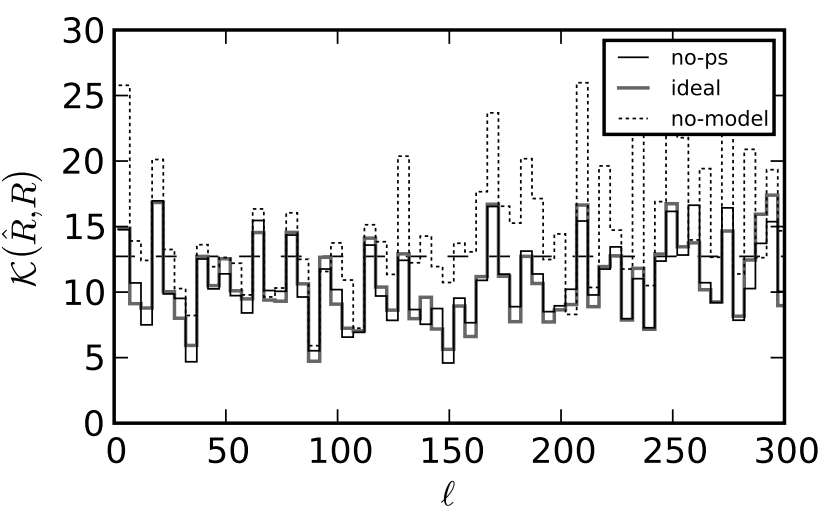

Fig. 6. Goodness-of-fit for the three point sources cases. For the reference case "no-ps", point sources have neither been including in the simulation, nor taken into account in the modelling. The mismatch criterion wander around its expectation value (horizontal dashed line). The "nomodel" case is a pessimistic situation where no effort has been made to model the point sources contribution, yielding a net increase of the mismatch criterion. The "ideal" case presents an optimistic situation where the exact contribution of the simulated point sources has been used to build the model.

ponent, which adjusts itself (via the values of its maximum likelihood parameters) to represent best the total foreground emission. The remaining part is responsible for the increase of the mismatch at high $\ell$. At the same time, the galactic estimation is twisted by the presence of point sources. This slightly increases the mismatch on large scales.

\subsection{Galactic foregrounds uncertainties}

We now investigate the impact on the above results of modifying somewhat the galactic emission. In particular, we check whether a space dependant curvature of the synchrotron spectral index, and modifications of the dust angular power spectrum, significantly change the error bars on $r$ obtained in the previous section. Those two modifications reflect two of the main uncertainties on diffuse foregrounds modelling discussed in Sect. 2.4. Although it is quite expectable that substantial variations of dust emission law can occur, modifications of the model in this sense would remain purely speculative and we choose to stick with simple modification of the (poorly constrained) dust emission level. Those two points are also representative of two different kinds of modelling errors: the first modification impacts the coherence of the emission across channels, while the second one illustrates the effect of an overall increase of the contamination level.

\subsubsection{Impact of synchrotron curvature}

As mentioned earlier on, the synchrotron emission law may not be perfectly described as a single power law per pixel, with a constant spectral index across frequencies. Steepening of the spectral index is expected in the frequency range of interest. As
Table 5. Influence of the running of the synchrotron spectral index on component separation in term of goodness of fit and $r$ estimates for the EPIC-2m design.

\begin{tabular}{ccccc}
\hline \hline$r$ & $\sigma_{r}$ & $\alpha$ & $r_{\text {est }}$ & $-\ln \mathcal{L}$ \\
\hline & & 0 & $9.78 \times 10^{-4}$ & 11.6 \\
0.001 & $1.8 \times 10^{-4}$ & 1 & $9.62 \times 10^{-4}$ & 11.5 \\
& & 3 & $1.06 \times 10^{-3}$ & 11.7 \\
\hline
\end{tabular}

Table 6. Influence of dust polarisation level on component separation.

\begin{tabular}{cccccc}
\hline \hline Experiments & $r$ & $r^{\text {origin }}$ & $r^{\text {pessim }}$ & $\sigma_{r}^{\text {origin }}$ & $\sigma_{r}^{\text {pessim }}$ \\
\hline Ground - based & 0.01 & $1.84 \times 10^{-2}$ & $1.69 \times 10^{-2}$ & $1.62 \times 10^{-2}$ & $1.62 \times 10^{-2}$ \\
EPIC - $2 \mathrm{~m}$ & 0.001 & $8.77 \times 10^{-4}$ & $8.77 \times 10^{-4}$ & $3.68 \times 10^{-4}$ & $3.61 \times 10^{-4}$ \\
\hline
\end{tabular}

this variation is related to the aging of cosmic rays, it should vary on the sky. Hence, the next level of sophistication in modelling synchrotron emission makes use of a (random) template map $C(\xi)$ to model the curvature of the synchrotron spectral index. We then produce simulated synchrotron maps as:

$S_{v}^{X}(\xi)=S_{v_{0}}^{X}(\xi)\left(\frac{v}{v_{0}}\right)^{\beta_{s}(\xi)+\alpha C(\xi) \log \left(v / v_{1}\right)}$

where $\alpha$ is a free parameter which allows to modulate the amplitude of the effect (as compared to Eq. (3)). The left panel of Fig. 7 illustrates the impact of the steepening on the synchrotron frequency scaling.

We now investigate whether such a modified synchrotron changes the accuracy with which $r$ can be measured. We decide, for illustrative purposes, to perform the comparison for EPIC$2 \mathrm{~m}$, and for $r=0.001$. Everything else, regarding the other emissions and the foreground model in SMICA, remains unchanged. Table 5 shows the results of this study in terms of goodness of fit and influence on the $r$ estimate. We observe no significant effect, which indicates that the foreground emission model of Eq. (17) is flexible enough to accommodate the variation of the synchrotron modelling. Even if we cannot test all possible deviation from the baseline PSM model, robustness against running of the spectral index remains a good indication that results are not overly model dependent.

\subsubsection{Level and power spectrum of dust emission}

Similarly, we now vary the model of dust emission and check how the main results of Sect. 4 are modified. Measurements give some constraints on dust emission on large scales, but smaller scales remain mostly unconstrained. Hence, we consider here a pessimistic extreme in which we multiply the large-scale level of the dust by a factor of two, and flatten the power spectrum from a nominal index of -2.5 to -1.9 . The power spectra corresponding to these two cases are shown in Fig. 7 (right panel).

Running the same component separation pipeline for the ground based and the EPIC-2m experiments at their detection 

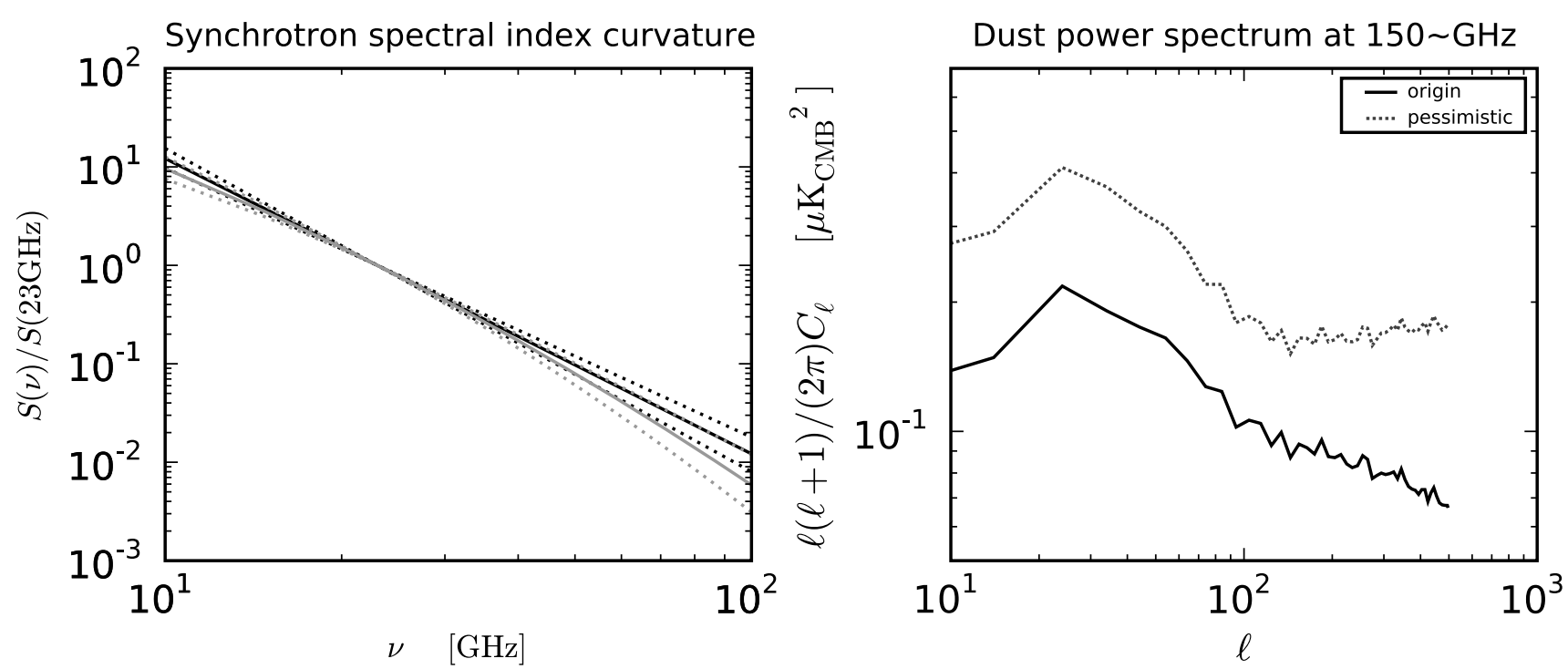

Fig. 7. Variations of the galactic foregrounds model. The left panel shows the dispersion of the synchrotron spectral index for the PSM model (in black) and the curved model (in gray). Solid lines present the frequency scaling for the mean values of the spectral index and dotted lines for its extremal values. The right panel shows the difference between the default power spectrum of dust polarisation B-modes at $150 \mathrm{GHz}$ as modelled by the PSM (solid curve) and a model assuming pessimistic values for the overall level and power spectrum index (dotted curve).

limit, we find only marginal changes in the measured values of $r$ (see Table 6). This result can be interpreted in the following way: as the noise of the experiment remains unchanged, the increased signal-to-noise ratio allows for a better constraint of the dust parameters. Component separation effectiveness depends mainly on the coherence of the component, rather than on its overall level.

\subsection{Error bar accuracy}

Estimates of the error derived from the FIM (Eq. (20)) are expected to be meaningful only if the model leading to the likelihood (Eq. (14)) holds. In particular we assume that processes can be modelled as Gaussian.

We first note that the FIM errors are reasonably compatible with the difference between input and measured $r$ values, which gives confidence that these error estimates are not obviously wrong. Nonetheless, we investigate this issue further, using Monte-Carlo studies to obtain comparative estimates of errors, with the EPIC-CS design. Table 7 gives, for two values of $r$ and for 100 runs of the SMICA pipeline in each case, the average recovered value of $r$, the average error as estimated from the Fisher matrix $\left\langle\sigma_{r}^{\text {FISHER }}\right\rangle$, and the standard deviation $\sigma_{r}^{\mathrm{MC}}$ of the measured values of $r$. For each of the Monte-Carlo runs, a new realisation of $\mathrm{CMB}$ and noise is generated. Simulated galactic foregrounds, however, remain unchanged.

Results show that the FIM approximation give estimates of the error in very good agreement with the MC result. Hence, the FIM estimate looks good enough for the purpose of the present paper, given the number of other potential sources of error and the computational cost of Monte-Carlo studies.

The Monte-Carlo study also allows to investigate the existence of a bias. For an input tensor to scalar ratio of 0.01 , we observe that the measured value of $r$ seems to be systematically low, with an average of $9.91 \times 10^{-3}$. This we interpret as resulting from a slight over-fitting of the data. Still this small bias does not dominate the error and we are more interested in noise dominated regime. The overall conclusion of this investigation
Table 7. Comparaison of the predicted error bar and the root mean squared error measured on 100 Monte Carlo realisations of EPIC-CS observation.

\begin{tabular}{cccc}
\hline \hline$r$ & $\langle r\rangle^{a}$ & $\left\langle\sigma_{r}^{\mathrm{FISHER}}\right\rangle^{a}$ & $\sigma_{r}^{\mathrm{MC}}$ \\
\hline 0.01 & $9.91 \times 10^{-3}$ & $3.59 \times 10^{-4}$ & $3.49 \times 10^{-4}$ \\
0.001 & $1.05 \times 10^{-3}$ & $1.84 \times 10^{-4}$ & $1.84 \times 10^{-4}$ \\
\hline
\end{tabular}

of error bars is that the errors estimated by the FIM are reasonably representative of the measurement error.

\subsection{Other cosmological parameters}

The main conclusions of this study are mostly independent of the value of all cosmological parameters except $\tau$. Within present uncertainties indeed, only the value of the reionisation optical depth $\tau$, which drives the amplitude and position of the reionisation bump, is critical for our estimations (Colombo et al. 2008). Lower $\tau$ means less accurate measurement of $r$, and higher $\tau$ better measurement of $r$. Here we choose a rather conservative value of $\tau=0.07$ in agreement with the last measurements from WMAP (Dunkley et al. 2008b,c). The value of $\tau$, however, should affect mainly low resolution and noisy experiments, for which most of the information comes from the lowest frequency "reionisation" bump in the B-mode spectrum.

Another issue is that we assume the value of $\tau$ and $n_{t}$ (and, to a less extent, the value of all other cosmological parameters) to be perfectly known (setting the shape of the B-mode power spectrum). In fact, uncertainties on all cosmological parameters imply that the shape will be known only approximately, and within a certain framework. Such uncertainties will have to be taken into account in the analysis of a real-life data set. Our SMICA pipeline can be adapted to do this, provided we know the uncertainties on the cosmological parameter set. A MonteCarlo approach, in which we assume, for each SMICA run, a Bmode power spectrum from one of the possible cosmological parameter sets, will permit to propagate the uncertainties onto the 
measurement of $r$. We expect, however, that this additional error will be significantly smaller than that due to the experimental noise.

\section{Conclusion}

In this paper, we presented an investigation of the impact of foregrounds on the measurement of the tensor to scalar ratio of primordial perturbations. The measurement of $r$ is based on the (simulated) observation of the B-mode polarisation of the cosmic microwave background by various instruments, either in preparation or planned for the future: the Planck space mission, a ground-based experiment of the type of $\mathrm{C} \ell$ over, and several versions of a possible dedicated space mission.

Foreground contamination is modelled and simulated using the present development version (v1.6.4) of the PSM. Our main analysis considers the contribution from diffuse polarised emission (from the galactic interstellar medium modelled as a mixture of synchrotron emission and thermal emission from dust) and from instrumental noise. The impact of more complicated galactic foreground emission, and of point sources and lensing, is investigated in a second step.

Our approach uses the SMICA component separation method on maps of B-modes alone. The method is robust with respect to specifics of foreground emission, because it does not rely on an accurate representation of foreground properties. That last point is demonstrated by varying the input foreground sky, and comparing results obtained with different inputs, without changing the analysis pipeline.

It is shown that for $r$ at the level of $r \simeq 0.1$, Planck could make a meaningful $(3 \sigma)$ detection from B-modes alone. The final sensitivity of Planck for measuring $r$ may be better than what is achieved here, as a significant part of the constraining power on $r$ should also come from EE/TE for high $r$. This has not been investigated in the present paper, which is more focussed on the measurement of low values of $r$ (not achievable with Planck). With the various EPIC mission designs, one could achieve detections at levels of 4-8 $\sigma$ for $r=10^{-3}$.

For full-sky, multi-frequency space missions, dealing with foregrounds in harmonic space results in a loss of sensitivity by a factor 3 to 4, as compared to what would be achievable without foregrounds, even if the covariance of foreground contaminants is known. The SMICA pipeline allows to achieve performances almost as good (within a factor 1.5), which demonstrates the effectiveness of the blind approach, but is still significantly worse (factor 3-5) than if there were no foregrounds at all. The loss of sensitivity is probably due in part to insufficient localisation in pixel space, which results in suboptimality of the estimator. This could (at least in principle) be improved with a localised processing.

For the most ambitious EPIC space mission, we find that our main conclusions are not modified significantly when taking into account the contamination of primordial B-modes by extragalactic point sources, by gravitational lensing, or when simulating a more complicated galactic emission. In contrast, we find that the measurement of $r$ from the ground with few frequency channels can be severely compromised by foregrounds, even in clean sky regions.

The joint analysis of such ground-based data together with those from less sensitive experiments covering a wider frequency range, such as the Planck data, permits to improve the constraints on $r$. Still, the result from a combined analysis of Planck and of a small patch observed from the ground at few frequencies cannot match what is obtained using sensitive measurements on the whole frequency range.

This makes a strong case for sensitive multi-frequency observations, and thus probably also for a space mission, as observations from the ground are severely limited (in frequency coverage) by atmospheric absorption and emission. This conclusion is further supported by the fact that a space mission mapping the same clean region (about 1\% of the sky), but with the full frequency range allowed by the absence of atmosphere, makes it possible to deal with diffuse foregrounds very efficiently.

Such a deep field mission would, in that respect, outperform a comparable full-sky experiment. The results obtained in the present study, however, do not permit to conclude whether a full sky or a deep field mission would ultimately perform better. A strategy based on the observation of a small patch seems to offer better prospects for measuring $r$ with an harmonic-space based version of SMICA, but also seems to be more impacted by smallscale contamination than all-sky experiments, and is in particular quite sensitive to the lensing effect. Further developments of the component separation pipeline could improve the processing of both types of datasets.

As a final comment, we would like to emphasise that the present study designs, implements effectively, and tests thoroughly on numerous simulations a component separation method for measuring $r$ with $\mathrm{CMB}$ B-modes which do not rely on a physical model of foreground emission. The method is shown to be robust against complicated foregrounds (pixel-dependent and running synchrotron spectral index, multitemplate dust emission, polarised point sources and lensing). It is also shown to provide reliable errors bars on $r$ by comparing analytical error bars (from the FIM) to estimates obtained from Monte-Carlo simulations. Although more work is needed for the optimal design of the next B-mode experiment, our results demonstrate that foregrounds can be handled quite effectively, making possible the measurement of $r$ down to values of 0.001 or better, at the $5-6 \sigma$ level.

Certainly, next steps will require fully taking into account small-scale contaminants, partial sky coverage effects, and probably some instrumental effects in addition to diffuse foregrounds. For this level of detail, however, it would be mandatory to refine as well the diffuse foreground model, using upcoming sensitive observations of the sky in the frequency range of interest and on both large and small scales. Such data will become available soon with the forthcoming Planck mission.

Acknowledgements. The authors acknowledge the use of the PSM, developed by the Component Separation Working Group (WG2) of the Planck Collaboration. The HEALPix package (Górski et al. 2005) was used for the derivation of some of the results presented in this paper. M.B. would like to thank USC for hospitality during the Spring 2008 and Marc-Antoine Miville-Deschênes for sharing his expertise on galactic foreground modelling. EP is an NSF-ADVANCE fellow (AST-0649899) also supported by NASA grant NNX07AH59G and Planck subcontract 1290790. J.D., J.F.C. and M.L.J. were partially supported by the ACI "Astro-Map" grant of the French ministry of research to develop the SMICA component separation package used here.

Partial support for EP and MB in carrying out this research was provided by the Jet Propulsion Laboratory, California Institute of Technology, under a contract with the National Aeronautics and Space Administration and funded through the Director's Research and Development Fund Program. (SURP award 1314616)"

\section{References}

Amblard, A., Cooray, A., \& Kaplinghat, M. 2007, Phys. Rev. D, 75, 083508 Audit, E., \& Simmons, J. F. L. 1999, MNRAS, 305, L27

Aumont, J., \& Macías-Pérez, J. F. 2007, MNRAS, 376, 739

Battistelli, E. S., Rebolo, R., Rubiño-Martín, J. A., et al. 2006, ApJ, 645, L141 
Baumann, D., \& Peiris, H. V. 2008, ArXiv e-prints

Baumann, D., Jackson, M. G., Adshead, P., et al. 2008, ArXiv e-prints

Bennett, C. L., Hill, R. S., Hinshaw, G., et al. 2003, ApJS, 148, 97

Benoît, A., Ade, P., Amblard, A., et al. 2004, A\&A, 424, 571

Bock, J., Cooray, A., Hanany, S., et al. 2008, ArXiv e-prints

Cardoso, J.-F., Le Jeune, M., Delabrouille, J., Betoule, M., \& Patanchon, G. 2008, ArXiv e-prints, 803

Challinor, A., Chon, G., Hivon, E., Prunet, S., \& Szapudi, I. 2003, New Astron. Rev., 47, 995

Colombo, L. P. L., \& Pierpaoli, E. 2008, ArXiv e-prints

Colombo, L. P. L., Pierpaoli, E., \& Pritchard, J. R. 2008, ArXiv e-prints

de Oliveira-Costa, A., Tegmark, M., Davies, R. D., et al. 2004, ApJ, 606, L89

de Zotti, G., Ricci, R., Mesa, D., et al. 2005, A\&A, 431, 893

Delabrouille, J., Cardoso, J.-F., \& Patanchon, G. 2003, MNRAS, 346, 1089

Delabrouille, J., Cardoso, J., Le Jeune, M., et al. 2008, ArXiv e-prints

Delabrouille, et al. 2009, in preparation

Désert, F.-X., Boulanger, F., \& Puget, J. L. 1990, A\&A, 237, 215

Draine, B. T., \& Fraisse, A. A. 2008, ArXiv e-prints

Draine, B. T., \& Lazarian, A. 1998, ApJ, 508, 157

Dunkley, J., Amblard, A., Baccigalupi, C., et al. 2008a, ArXiv e-prints

Dunkley, J., Komatsu, E., Nolta, M. R., et al. 2008b, ArXiv e-prints, 803

Dunkley, J., Spergel, D. N., Komatsu, E., et al. 2008c, ArXiv e-prints

Finkbeiner, D. P. 2004, ApJ, 614, 186

Finkbeiner, D. P., Davis, M., \& Schlegel, D. J. 1999, ApJ, 524, 867

Fosalba, P., Lazarian, A., Prunet, S., \& Tauber, J. A. 2002, ApJ, 564, 762

Giardino, G., Banday, A. J., Górski, K. M., et al. 2002, A\&A, 387, 82

Gold, B., Bennett, C. L., Hill, R. S., et al. 2008, ArXiv e-prints, 803

Górski, K. M., Hivon, E., Banday, A. J., et al. 2005, ApJ, 622, 759

Haslam, C. G. T., Salter, C. J., Stoffel, H., \& Wilson, W. E. 1982, A\&AS, 47, 1

Hirata, C. M., \& Seljak, U. 2003, Phys. Rev. D, 67, 043001

Hivon, E., Górski, K. M., Netterfield, C. B., et al. 2002, ApJ, 567, 2

Hu, W., \& White, M. 1997, New Astron., 2, 323

Hu, W., Hedman, M. M., \& Zaldarriaga, M. 2003, Phys. Rev. D, 67, 043004

Jonas, J. L., Baart, E. E., \& Nicolson, G. D. 1998, MNRAS, 297, 977

Kamionkowski, M., \& Kosowsky, A. 1998, Phys. Rev. D, 57, 685

Kamionkowski, M., Kosowsky, A., \& Stebbins, A. 1997, Phys. Rev. Lett., 78, 2058

Kaplan, J., \& Delabrouille, J. 2002, in Astrophysical Polarized Backgrounds, ed.

S. Cecchini, S. Cortiglioni, R. Sault, \& C. Sbarra, AIP Conf. Ser., 609, 209

Kesden, M., Cooray, A., \& Kamionkowski, M. 2003, Phys. Rev. D, 67, 123507
Knox, L., \& Song, Y.-S. 2002, Phys. Rev. Lett., 89, 011303

Kovac, J. M., Leitch, E. M., Pryke, C., et al. 2002, Nature, 420, 772

Lazarian, A. 2007, J. Quant. Spec. Rad. Trans., 106, 225

Lazarian, A., \& Finkbeiner, D. 2003, New Astron. Rev., 47, 1107

Lewis, A., \& Challinor, A. 2006, Phys. Rep., 429, 1

Li, A., \& Draine, B. T. 2001, ApJ, 554, 778

Macellari, N., Pierpaoli, E., Dickinson, C., \& Vaillancourt, J. 2008, in prep.

Miville-Deschênes, M., Ysard, N., Lavabre, A., et al. 2008, ArXiv e-prints, 802

Moudden, Y., Cardoso, J., Starck, J., \& Delabrouille, J. 2004, ArXiv Astrophysics e-prints

North, C. E., Johnson, B. R., Ade, P. A. R., et al. 2008, ArXiv e-prints

Page, L., Hinshaw, G., Komatsu, E., et al. 2007, ApJS, 170, 335

Peiris, H. V., Komatsu, E., Verde, L., et al. 2003, ApJS, 148, 213

Pierpaoli, E., \& Perna, R. 2004, MNRAS, 354, 1005

Platania, P., Burigana, C., Maino, D., et al. 2003, A\&A, 410, 847

Reich, P., \& Reich, W. 1986, A\&AS, 63, 205

Ricci, R., Prandoni, I., Gruppioni, C., Sault, R. J., \& De Zotti, G. 2004, A\&A, 415, 549

Rosset, C., Yurchenko, V. B., Delabrouille, J., et al. 2007, A\&A, 464, 405

Rybicki, G. B., \& Lightman, A. P. 1979, Radiative processes in astrophysics (New York: Wiley-Interscience), 393

Sazonov, S. Y., \& Sunyaev, R. A. 1999, MNRAS, 310, 765

Schlegel, D. J., Finkbeiner, D. P., \& Davis, M. 1998, ApJ, 500, 525

Seljak, U., \& Zaldarriaga, M. 1997, Phys. Rev. Lett., 78, 2054

Seto, N., \& Pierpaoli, E. 2005, Phys. Rev. Lett., 95, 101302

Sievers, J., \& CBI Collaboration. 2005, in BAAS, 37, 1329

Smith, K. M., \& Zaldarriaga, M. 2007, Phys. Rev. D, 76, 043001

Smith, K. M., Cooray, A., Das, S., et al. 2008, ArXiv e-prints

Strong, A. W., Moskalenko, I. V., \& Ptuskin, V. S. 2007, Ann. Rev. Nucl. Part. Sci., 57, 285

Tegmark, M., de Oliveira-Costa, A., \& Hamilton, A. J. 2003, Phys. Rev. D, 68, 123523

Tucci, M., Martínez-González, E., Toffolatti, L., González-Nuevo, J., \& De Zotti, G. 2004, MNRAS, 349, 1267

Tucci, M., Martínez-González, E., Vielva, P., \& Delabrouille, J. 2005, MNRAS, 360,935

Verde, L., Peiris, H. V., \& Jimenez, R. 2006, J. Cosmol. Astro-Part. Phys., 1, 19

Zaldarriaga, M., \& Seljak, U. 2000, ApJS, 129, 431

Zaldarriaga, M., Seljak, U., \& Bertschinger, E. 1998, ApJ, 494, 491 


\section{Appendix A: Parameterisation the foreground component and choice of a mask}

In this appendix, we discuss in more detail the dimension $D$ of matrix used to represent the covariance of the total galactic emission, and the choice of a mask to hide regions of strong galactic emission for the estimation of $r$ with SMICA.

\section{A.1. Dimension $D$ of the foreground component}

First, we explain on a few examples the mechanisms which set the rank of the foreground covariance matrix, to give an intuitive understanding of how the dimension $D$ of the foregrounds component used in SMICA to obtain a good model of the data. Let us consider the case of a "perfectly coherent" physical process, for which the total emission, as a function of sky direction $\xi$ and frequency $v$, is well described by a spatial template multiplied by a pixel-independent power law frequency scaling:

$S_{v}(\xi)=S_{0}(\xi)\left(\frac{v}{v_{0}}\right)^{\beta}$.

The covariance matrix of this foreground will be of rank one and $\mathrm{R}^{\mathrm{S}}=\left[\mathbf{A A}^{\dagger} \operatorname{var}\left(S_{0}\right)\right]$, with $A_{f}=\left(\frac{v_{f}}{v_{0}}\right)^{\beta}$. Now, if the spectral index $\beta$ fluctuates on the sky, $\beta(\xi)=\beta+\delta \beta(\xi)$, to first order, the emission at frequency $v$ around $v_{0}$ can be written:

$S_{v}(\xi) \approx S_{0}(\xi)\left(\frac{v}{v_{0}}\right)^{\beta}+S_{0}(\xi)\left(\frac{v}{v_{0}}\right)^{\beta} \delta \beta(\xi)\left(\frac{v-v_{0}}{v_{0}}\right)$.

This is not necessarily the best linear approximation of the emission, but supposing it holds, the covariance matrix of the foreground will be of rank two (as the sum of two correlated rank 1 processes). If the noise level is sufficiently low, the variation introduced by the first order term of Eq. (A.2) becomes truly significant, we can't model the emission by a mono-dimensional component as in Eq. (A.1).

In this work, we consider two processes, synchrotron and dust, which are expected to be correlated (at least by the galactic magnetic field and the general shape of the galaxy). Moreover, significant spatial variation of their emission law arises (due to cosmic aging, dust temperature variation ...), which makes their emission only partially coherent from one channel to another. Consequently, we expect that the required dimension $D$ of the galactic foreground component will be at least 4 as soon as the noise level of the instrument is low enough.

The selection of the model can also be made on the basis of a statistical criterion. For example, Table A.1 shows the Bayesian information criterion (BIC) in the case of the EPIC-2m experiment $(r=0.01)$ for 3 consecutive values of $D$. The BIC is a decreasing function of the likelihood and of the number of parameter. Hence, lower BIC implies either fewer explanatory variables, better fit, or both. In our case the criterion reads:

$B I C=-2 \ln \mathcal{L}+k \ln \sum_{q} w_{q}$

where $k$ is the number of estimated parameters and $w_{q}$ the effective number of modes in bin $q$. Taking into account the redundancy in the parameterisation, the actual number of free parameters in the model is $1+F \times D+Q D(D+1) / 2-D^{2}$. However, we usually prefer to rely on the inspection of the mismatch in every bin of $\ell$, as some frequency specific features may be diluted in the global mismatch.
Table A.1. Bayesian information criterion of 3 models with increasing dimension of the galactic component for the EPIC-2m mission.

\begin{tabular}{ccc}
\hline \hline$D$ & $k$ & $B I C$ \\
\hline 3 & 376 & $1.15 \times 10^{4}$ \\
4 & 617 & $8.35 \times 10^{3}$ \\
5 & 916 & $1.15 \times 10^{4}$ \\
\hline
\end{tabular}

\section{A.2. Masking influence}

The noise level and the scanning strategy remaining fixed in the full-sky experiments, a larger coverage gives more information and should result in tighter constraints on both foreground and CMB. In practice, it is only the case up to a certain point, due to the non stationarity of the foreground emission. In the galactic plane, the emission is too strong and too complex to fit in the proposed model, and this region must be discarded to avoid contamination of the results. The main points governing the choice of an appropriate mask are the following:

- the covariance of the total galactic emission (synchrotron and dust polarised emissions), because of the variation of emission laws as a function of the direction on the sky, is never exactly modelled by a rank $D$ matrix. However it is satisfactorily modelled in this way if the difference between the actual second order statistics of the foregrounds, and those of the rank $D$ matrix model, are indistinguishable because of the noise level (or because of cosmic variance in the empirical statistics). The deviation from the model is more obvious in regions of strong galactic emission, hence the need for a galactic mask. The higher the noise, the smaller the required mask;

- SMICA provides a built-in measure of the adequacy of the model, which is the value of the spectral mismatch. If too high, the model under-fits the data, and the dimension of the foreground model (or the size of the mask) should be increased. If too low, the model over-fits the data, and $D$ should be decreased;

- near full sky coverage is better for measuring adequately the reionisation bump;

- the dimension of the foreground component must be smaller than the number of channels.

If the error variance is always dominated by noise and cosmic variance, the issue is solved: one should select the smaller mask that gives a good fit between the model and the data to minimise the mean squared error and keep the estimator unbiased.

If, on the other hand, the error seems dominated by the contribution of foregrounds, which is, for example, the case of the EPIC-2m experiment for $r=0.001$, the tradeoff is unclear and it may happen that a better estimator is obtained with a stronger masking of the foreground contamination. We found that it is not the case. Table A.2 illustrates the case of the EPIC-2m experiment with the galactic cut used in Sect. 4 and a bigger cut. Although the reduction of sensitivity is slower in the presence of foreground than for the noise dominated case, the smaller mask still give the better results. We may also recall that the expression (7) of the likelihood is an approximation for partial sky coverage. The scheme presented here thus may not give fully reliable results when masking effects become important. 
Table A.2. Estimation of the tensor to scalar ratio with two different galactic cuts in the EPIC-2m experiment.

\begin{tabular}{ccccc}
\hline \hline$r$ & $r^{\text {est }}$ & $\sigma_{r}^{\text {FISHER }}$ & $\sigma_{r}^{\text {no-fg }}$ & $f_{\text {sky }}$ \\
\hline 0.001 & $1.01 \times 10^{-3}$ & $1.60 \times 10^{-4}$ & $5.25 \times 10^{-5}$ & 0.87 \\
0.001 & $1.01 \times 10^{-3}$ & $1.68 \times 10^{-4}$ & $5.72 \times 10^{-5}$ & 0.73 \\
\hline
\end{tabular}

\section{Appendix B: Spectral mismatch}

Computed for each bin $q$ of $\ell$, the mismatch criterion, $w_{q} K\left(\widehat{\mathrm{R}}_{q}, \mathrm{R}_{q}\left(\theta^{*}\right)\right)$, between the best-fit model $\mathrm{R}_{q}\left(\theta^{*}\right)$ at the point of convergence $\theta^{*}$, and the data $\widehat{\mathrm{R}}_{q}$, gives a picture of the goodness of fit as a function of the scale. Black curves in Figs. B.1 and B.2 show the mismatch criterion of the best fits for Planck and EPIC designs respectively. When the model holds, the value of the mismatch is expected to be around the number of degrees of freedom (horizontal black lines in the figures). We can also compute the mismatch for a model in which we discard the CMB contribution $w_{q} K\left(\widehat{\mathrm{R}}_{q}, \mathrm{R}_{q}\left(\theta^{*}\right)-\mathrm{R}_{q}^{\mathrm{CMB}}\left(r^{*}\right)\right)$. Gray curves in Figs. B.1 and B.2 show the mismatch for this modified model. The difference between the two curves illustrates the "weight" of the CMB component in the fit, as a function of the scale.

Figure B. 1 shows the results for Planck for $r=0.3$ and 0.1 . The curves of the difference plotted in inclusion illustrate the predominance of the reionisation bump. In Fig. B.2, we plot the difference curve on the bottom panels for the three experiments for $r=0.01$ and $r=0.001$. They illustrate clearly the difference of sensitivity to the peak between the EPIC-LC design and the higher resolution experiments. In general it can be seen that no significant contribution to the $\mathrm{CMB}$ is coming from scales smaller than $\ell=150$.

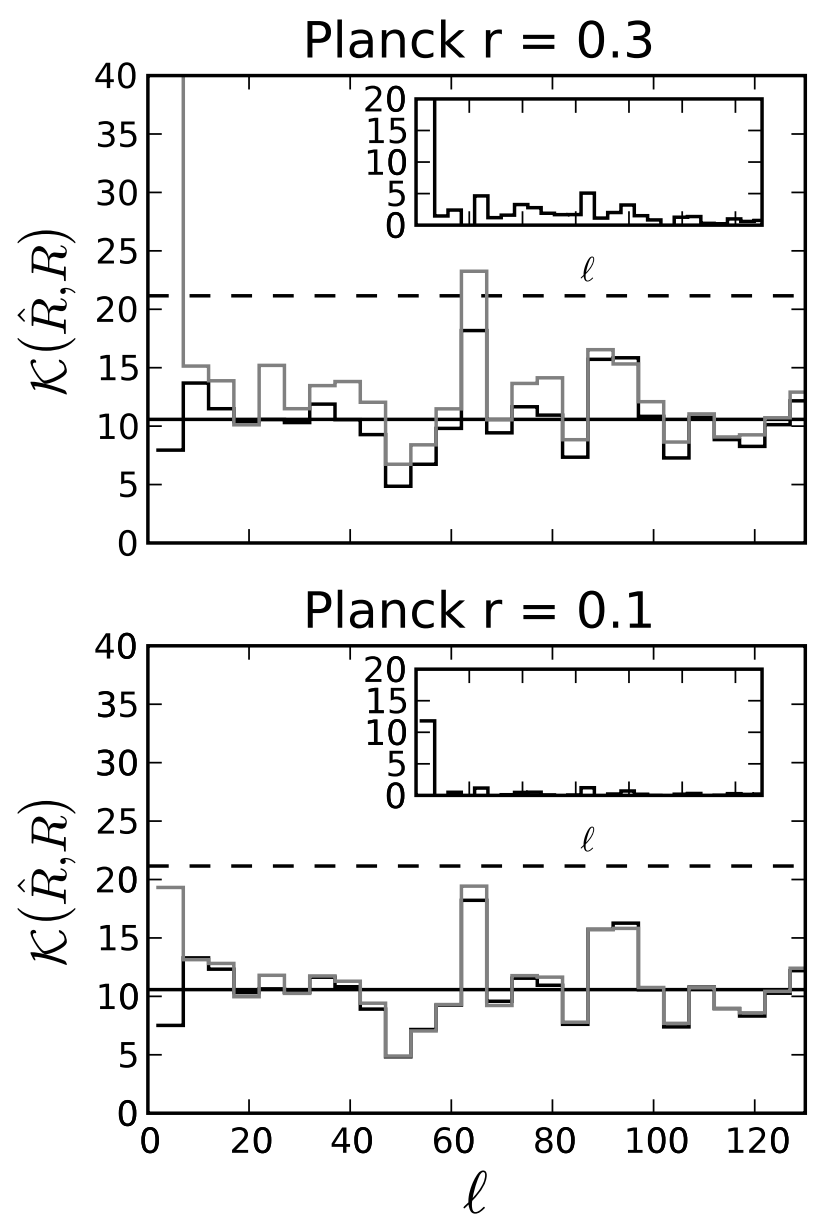

Fig. B.1. Those plots present the distribution in $\ell$ of the mismatch criterion between the model and the data for two values of $r$ for PLANCK. On the grey curve, the mismatch has been computed discarding the CMB contribution from the SMICA model. The difference between the two curves, plotted in inclusion, illustrates somehow the importance of the $\mathrm{CMB}$ contribution to the signal. 
M. Betoule et al.: $T / S$ measurements in the presence of foregrounds, Online Material $p 3$
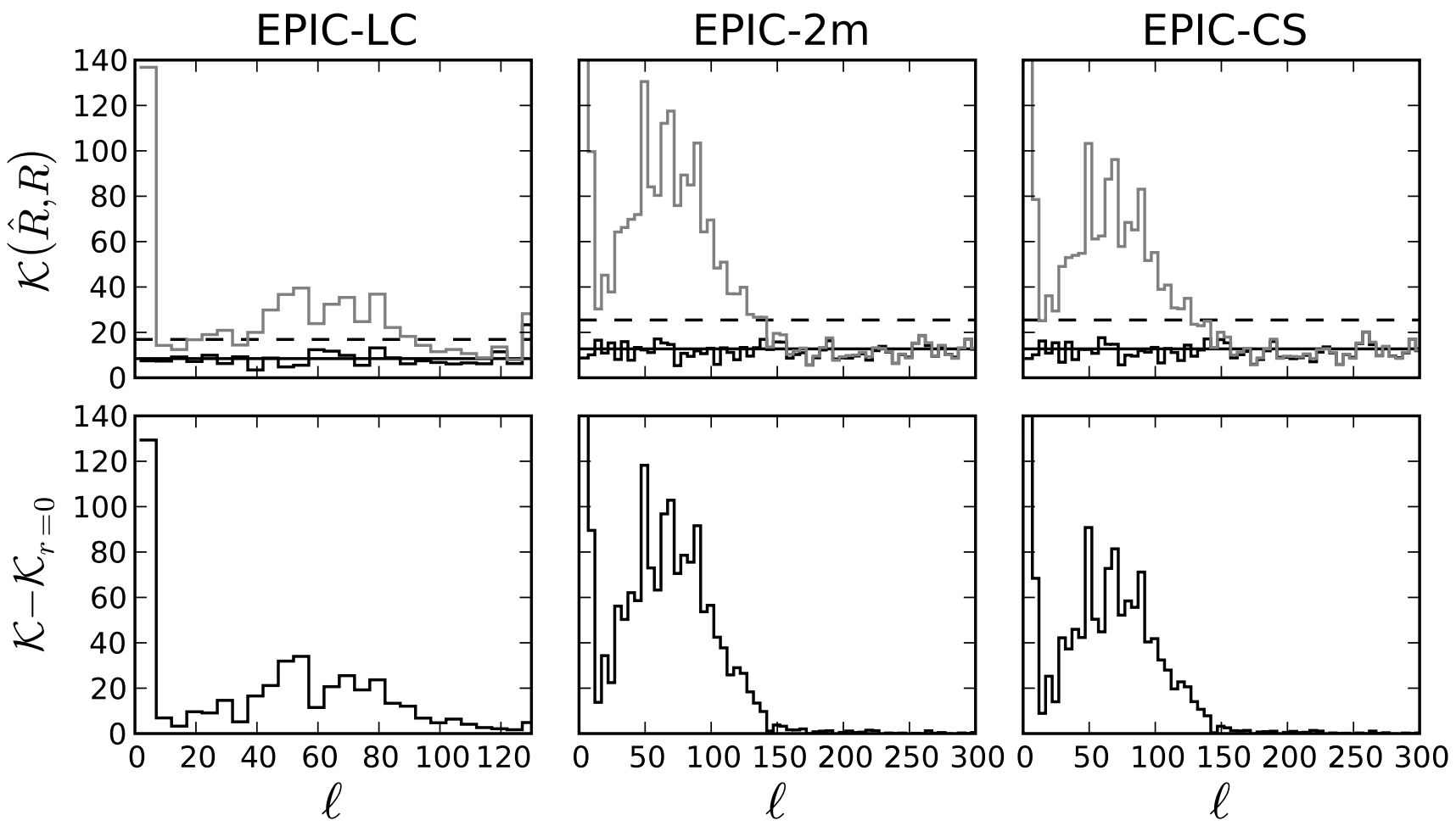

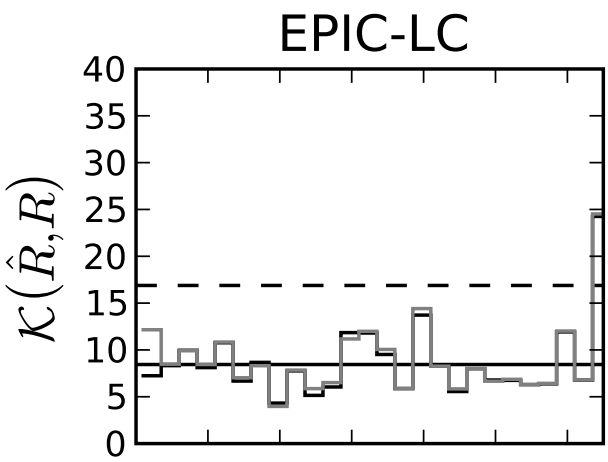

EPIC-2m
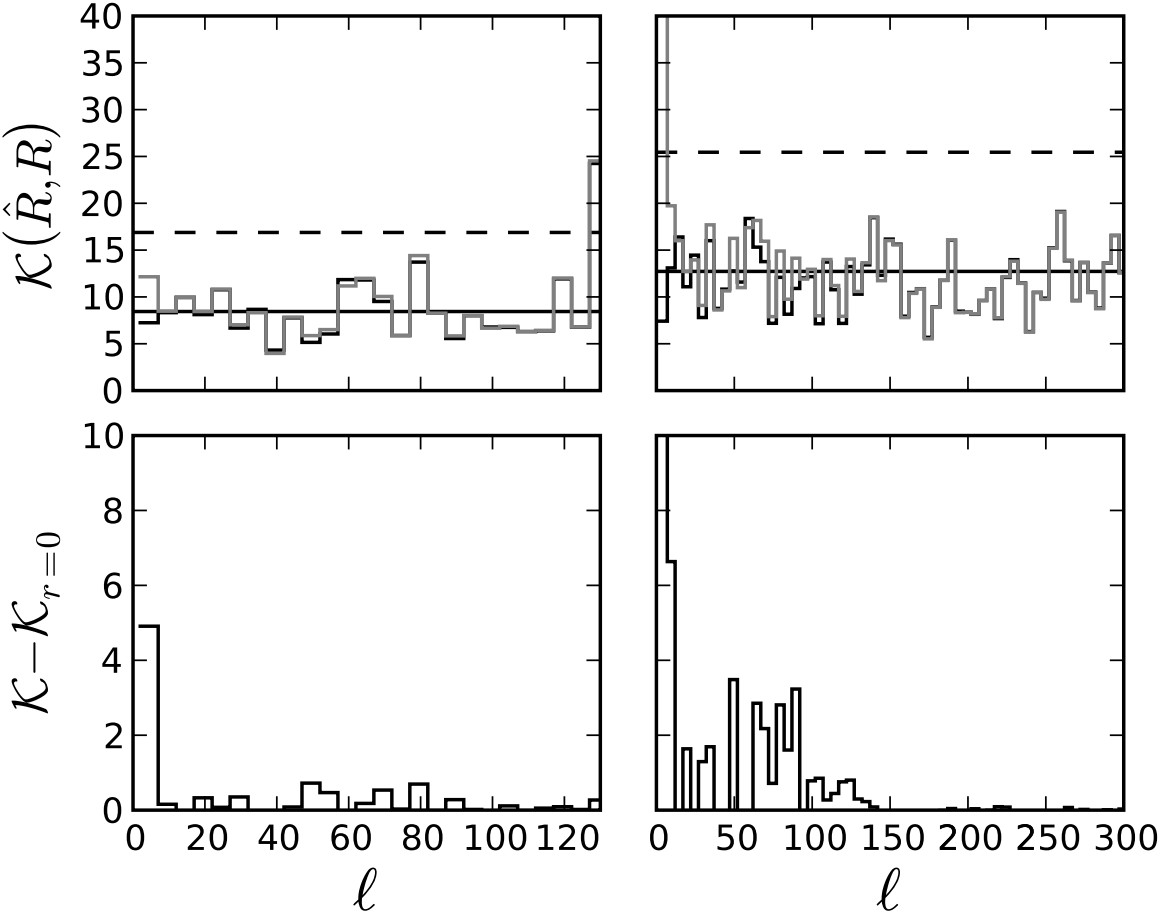

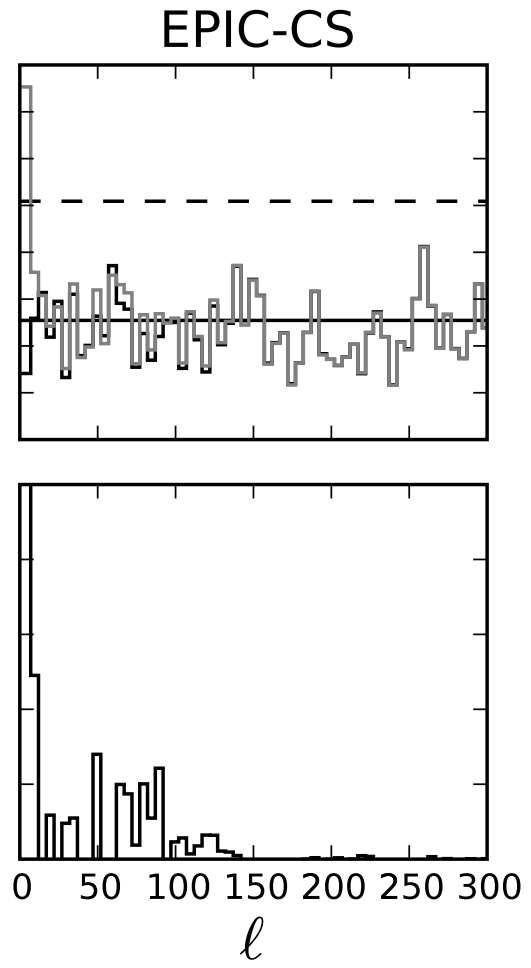

Fig. B.2. Mismatch criterion for $r=0.01$ (top) and $r=0.001$ (bottom). In each plot, the top panel shows the mismatch criterion between the best fit model and the data (black curve) and the best fit model deprived from the CMB contribution and the data (gray curve). Solid and dashed horizontal lines show respectively the mismatch expectation and 2 times the mismatch expectation. The difference between the gray and the black curve is plotted in the bottom panel and gives an idea of the significance of the CMB signal in each bin of $\ell$. 